\title{
MORBILIDAD Y MORTALIDAD EN EL INSTITUTO MATERNO INFANTIL "CONCEPCION VILLAVECES DE ACOSTA"

\author{
Bogotá
}

\author{
Años de 1960, 1961, 1962, 1963 y 1964 \\ Doctor Rafael López-Ruiz*
}

El Instituto Materno Infantil forma con el Hospital propiamente dicho el conjunto denominado Establecimientos Hospitalarios de San Juan de Dios.

Juntas Instituciones constituyen actividades de la Beneficencia de Cundinamarca, ambas son interindependientes y cada una absolutamente autónoma en sus funciones científicas y administrativas.

Por gentilísima y obligante invitación de la Revista de Obstetricia y Ginecología presentamos, de nuestros trabajos anteriores sobre Estadística de los Establecimientos Asistenciales de San Juan de Dios, solamente aquello que atañe directa y particularmente al Instituto Materno Infantil "Concepción Villaveces de Acosta".

Recordamos que la fuente primera de datos en toda Institución Hospitalaria es la Historia Clínica, la cual, para ser en realidad eficiente en su co-

* Jefe de Estadística Científica de los Establecimientos Asistenciales de San Juan de Dios, metido debe tener las siguientes cualidades: bien presentada es decir, pulcra y ordenada, clara es decir, escrita en caracteres legibles (mejor mecanografiada), completa o sea con todos los informes referentes a la identificación del paciente, a las fechas de entrada y de salida, a la causa o motivo de ésta $y$, con todas, absolutamente todas las indicaciones científicas o sea con todos los diagnósticos y las anotaciones de tratamientos médicos o quirúrgicos. La Historia debe ser también oportuna, vale decir, que su envío del servicio de donde proviene a la Oficina Central de Estadística en donde se tabulan todos los datos, debe hacerse, a más tardar, dentro de las 48 horas posteriores a la salida del paciente. En algunos establecimientos el reglamento es tan estricto y severo a este respecto que exige, so pena de sanciones, la mencionada entrega al cabo, inaplazable, de 24 horas. La necesidad absoluta de esta oportunidad es obvia por cuanto que toda historia demorada retarda los informes pertinentes a su época y obliga a hacer una estadística cronológica- 
mente inexacta, ya que ocasiona la contabilización de DATOS de un mes dentro del conjunto de otra mensualidad.

Siendo la Historia Clínica la representación pasada, presente y futura del paciente; la realización intachable de este documento, su cuidado, presentación y entrega final corresponden, en obligación ineludible, a todo el servicio; desde el profesor jefe hasta la última enfermera, pasando por asistentes, consultantes, residentes, internos y religiosas viligantes de sala o personal laico con las mismas obligaciones.

Nos hemos permitido insistir en estos puntos porque hay establecimientos en los cuales la Historia Clínica es un documento baladí, mal llevado, descuidado en todas sus líneas generales, frente al cual desfilan con olímpico desprecio, científicos y auxiliares como haciendo ver que su importancia personal no puede descender hasta "ese pequeño detalle" de la Historia Clínica.

Magno error este porque "la Historia Clínica es el testimonio fehaciente de todo aquello que el médico sabe y puede $\mathrm{y}$, por tanto, de todo aquello que el paciente recibe como atención científica eficiente y oportuna ayuda humanitaria" (Definición dada por el Delegado colombiano a la primera Convención Interamericana de Hospitales (La Habana, noviembre, 1956) y aceptada por unanimidad). De toda la inmensa realidad de la existencia de la Historia Clínica se deduce, sin ambajes, que los médicos, deben fomentar solícitamente ese testimonio de su saber y de sus actuaciones para con el paciente, y que todo descuido, desaire o pretensión negativa al respecto es, no solamente una automutilación, sino una negligencia profesional grave que muchas veces puede convertirse en acusación con testimonio de ignorancia y culpabilidad por negligencia. Entre la conciencia de responsabilidad del médico debe estar, firmemente engastada, la de la Historia Clínica.

Creemos en verdad que entre las enseñanzas básicas de los primeros años de estudios médicos debe figurar la de la realización y cuidado de la Historia Clínica. Despertando en los médicos desde los primeros años de su labor intelectual hipocrática la conciencia de la responsabilidad que acabamos de mencionar, las Facultades de Medicina harían una labor docente digna de tal objetivo ya que subsanaría los graves problemas que en relación con la Historia Clínica han contemplado las Asociaciones de Facultades y las de Hospitales.

\section{Acervo científico}

Un material de 150.000 historias clínicas en la actualidad, primordial elemento para la investigación, ha sido y sigue siendo puesto a la orden de los autores según los requerimientos de selección, precisión y oportunidad por la Sección de Codificación y Archivo que cataloga todos los diagnósticos según el sistema "standard" — patrónde la Asociación Médica Americana, cruzado con el de la O.M.S. (Organización Mundial de la Salud). El primero; permite el hallazgo seguro de los documentos relacionados con cualquiera topografía o localización de enfermedad y con no importan cual etiología 
diagnosticada. El segundo; Clasificación Estadística de Enfermedades y causas de defunción separa todas las dolencias, desde las infecciones hasta los traumatismos en 17 grupos bien especificados. Ambos sistemas que, cruzados, se vienen emp'eando en los Establecimientes Asistenciales de San Juan de Dios desde 1952 tiene en la Bibioteca de Historias Clínicas un acervo de incalculable valor investigativo cientíico y asistencial que está siempre a la disposición de todos aquellos que se dediquen a la búsqueda y a la conclusión fundamentadas en documentos hospitalarios pertinentes.

\section{Estadisticas de morbilidad, mortalidad $y$ movimiento asistencial}

Para uno y otro de los dos primeros capítulos enumerados utilizamos los grupos patológicos estadísticos de la $\mathrm{O}$. M. S. Este procedimiento nos facilita presentar un cuadro sinóptico perfectamente circunscrito. No vamos a detallar cada uno de los 999 renglones comprendidos entre los XVII grupos porque tales renglones tienen subdivisiones decimales y este conjunto de dígitos nos llevaría a realizar algo así como un diccionario de morbilidad y de mortalidad trabajo heroico y de inutilidad actual.

El objetivo estadístico lo creemos plenamente logrado condensando en totales generales las causas de muerte y los diagnósticos de morbilidad por grupos I a XVII O.M.S., dando los porcentajes respectivos e ilustrando cada capítulo con cuadros aritméticos y las gráficas correspondientes.

Debemos hacer observar que la unidad diagnóstico no significa, rigurosa- mente, la unidad paciente, o sea, que no se puede contar un enfermo por cada diagnóstico. Si esto fuera así habría una enorme discordancia entre el número de atendidos y la cantidad de afecciones contabilizadas. Sucede, en realidad que en muchas madres y en muchos niños se diagnostican, a la vez, tres, cuatro y hasta cinco afecciones. En efecto, en algunas madres las historias pueden llevar los siguientes diagnósticos: Aborto provocado, perforación uterina, pelviperitonitis, septicemia, anemia aguda. En los niños no es raro encontrar la siguiente acumulación patológica; labio leporino, paladar hendido, conjuntivitis, neumonía, deshidratación.

En el Instituto Materno Infantil la morbilidad no es, desde luego sino una; Obstétrica y ella toda está comprendida en la Clase XI de las ya mencionadas XVII de la O.M.S. Sin embargo, como al lado del movimiento asistencial obstétrico hay siempre una atención dedicada a entidades patológicas propiamente dichas, presentamos la estadística de esa morbilidad agrupada en las casillas respectivas de la O.M.S. y para cada uno de los años 1960, 1961, 1962, 1963, y 1964 (cuadros A, B, C, D, E). En esos cuadros la Clase XV. O. M. S. que corresponde a enfermedades de la primera infancia no lleva como es obvio, anotación alguna, ya que todo lo referente a ese capítulo se halla en los cuadros de morbilidad y mortalidad infantiles.

Hemos creído interesante destacar y colocar aparte (tabla G), las toxemias (años 1960, 1961, 1962, 1963) y Observaciones año 1964, mostrándolas en 
relación de porcentaje con el número global de pacientes admitidas ù hospitalizadas para parto.

También presentamos la morbilidad y la mortalidad infantiles, (cuadros $\mathrm{F}$, $\mathrm{G}, \mathrm{H}, \mathrm{I}, \mathrm{J}, \mathrm{K}, \mathrm{L}, \mathrm{M}, \mathrm{O}, \mathrm{P}, \mathrm{Q})$ figuras 1-5 que son variadas y de análisis altamente interesante. Debemos hacer notar que en la clasificación O.M.S. todas las enfermedades de la primera infancia están encerradas en un solo grupo, el XV. Nosotros la hemos distribuído, para hacerlas especialmente objetivas y tangibles, entre los XVII capítiulos o subdivisiones, aplicando así a la patología infantil, precisamente, la selección general establecida por la O.M.S.

De todas maneras el estudio de la morbilidad en un Hospital es básico para hacer una segura planificación de sus servicios en relación con equipo, personal y costos y para contribuír al estudio nacional e internacional de las medidas preventivas que, como de todos es sabido, deben preceder las de la medicina curativa. Es redundante, entonces, decir la importancia de la estadística médica materno infantil ya que la protección de la madre y del niño es política a la cual ningún gobierno puede esquivarse. A este respecto vemos en los cuadros de frecuencias del Instituto Materno Infantil que las enfermedades más comunes son las respiratorias. Es nuestro deber hacer notar que la anoxia clasificada por la O.M.S. en el grupo XVI — síntomas, síndromes - la hemos colocado de manera arbitraria y talvez pensando en el papel dẹl oxígeno en la respiración, entre las enfermedades del sistema respiratorio propiamente dicho. De todas maneras, aclaramos, que en nuestros cómputos las enfermedades respiratorias singulares fueron siempre las más numerosas aun sin la adición de los casos de anoxia.

Continuando este rápido análisis de las frecuencias en la patología infantil vemos aparecer, después de los males respiratorios las afecciones infecciosas y en seguida las de la sangre y órganos hematopoyéticos; discrasias, hemólisis, hemorragias, incompatabilidades sanguíneas, etc. (años 1960, 61, 62, 63), en 1964 al lado de las respiratorias e infecciosas figuran especialmente las dermatosis y las enfermedades digestivas.

Los traumatismos obstétricos tienen bastante importancia en la morbilidad infantil como lo demuestran las cifras crecientes de 1960 a 1964. Es verdad que la asistencia también aumentó progresivamente en esos años.

La mortalidad no neonatal (cuadros $\mathrm{M}, \mathrm{Q}$, figuras 2, 4), indican preponderancia de las afecciones respiratorias como causa principal, luego llegan las de los sistemas circulatorio y digestivo. Las defunciones Neo-natales (cuadros $\mathrm{L}$, P, figura 3), muestran predominios similares. La morbilidad materna (cuadros A, B, C, D, E, figura 6) (años 1960, 61, 62, 63, y 64 respectivamente) señala preponderancia de las enfermedades infecciosas y para 1964 las luéticas. Las afecciones neoplásicas ocupan sitio bien visible y al lado de ellas las genito urinarias, en seguida resaltan las de los sistemas circulatorio y digestivo. Las defunçiones maternas 
que hemos agrupado en los cuadres $\mathrm{R}$, $\mathrm{RR}, \mathrm{S}, \mathrm{T}, \mathrm{U}$, con discriminación del número de la historia correspondiente, edades y causas de la defunción según la historia, muestra como causa principal las toxemias y las repercusiones consiguientes en el organismo de la embarazada. Las peritonitis consecutivas a infecciones post-maniobras abortivas son importantes y hay dos casos (1960, 61) en los cuales la T.B.C. pulmonar fue sindicada como causa principal. Nos permitimos, a este respecto llamar la atención sobre las observaciones relacionadas con toxemias y los coeficientes de mortalidad.

\section{Movimiento asistencial}

Los diferentes cuadros sinópticos $\mathrm{X}$, $\mathrm{X}^{2}, \mathrm{Y}$; figuras $7,8,9,10,11$, señalan un sensible aumento en relación con los años anteriores. Crecieron de manera notable; las consultas de Pediatría, los análisis de Laboratorio y el número de partos atendidos. En general puede decirse que hay una progresión creciente en el aumento de los ingentes servicios que presta el Instituto a la clase desvalida que solicita su atención. Nos permitimos hacer hincapié sobre los renglones; estancias, promedio diario, índice de ocupación de camas sobre todo en el año de 1964. El Cuadro Z del mes de junio de 1964 de los Servicios Pediátricos da una completa idea de la magnífica labor que allí se está realizando y de la manera como se hace $y$ se tabula la estadística correspondiente. 


\section{CUADRO A}

INSTITUTO MATERNO INFANTIL

MORBILIDAD MATERNA (NO OBSTETRICA) EN EL AÑO DE 1960

CLASIFICADA SEGUN LOS GRUPOS ESTADISTICOS DE LA O.M.S.

TOT AL: 1.233

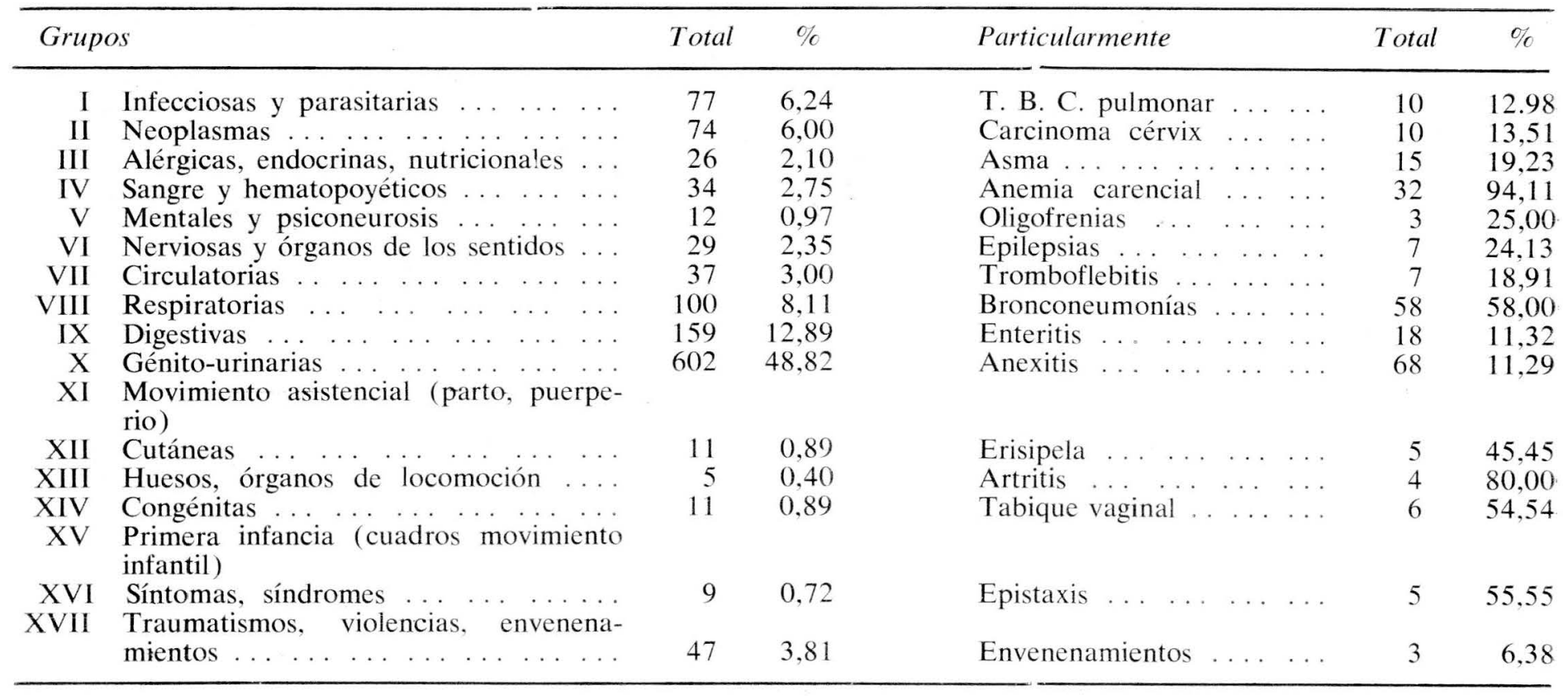


CUADRO B

INSTITUTO MATERNO INFANTIL

MORBILIDAD MATERNA (NO OBSTETRICA) EN EL AÑO DE 1961

CLASIFICACION SEGUN LOS GRUPOS ESTADISTICOS DE LA O.M.S.

TOT AL: 1.042

\begin{tabular}{|c|c|c|c|c|c|c|}
\hline \multicolumn{2}{|c|}{ Grupos } & \multirow{2}{*}{$\frac{\text { Total }}{94}$} & \multirow{2}{*}{$\frac{\%}{9,02}$} & Particularmente & \multirow{2}{*}{$\frac{\text { Total }}{10}$} & \multirow{2}{*}{$\frac{\%}{10,63}$} \\
\hline I & Infecciosas y parasitarias $\ldots \ldots \ldots$ & & & T. B. C. pulmonar ... . . & & \\
\hline II & 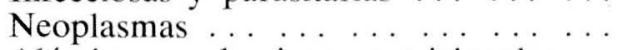 & 92 & 8,82 & Fibromas uterinos ... ... & 15 & 16,30 \\
\hline III & Alérgicas, endocrinas, nutricionales .. & 35 & 3,35 & Asma $\ldots \begin{array}{lllll} & \ldots & \ldots & \ldots & \ldots\end{array}$ & 19 & 54,28 \\
\hline IV & Sangre y hematopoyéticos $\ldots \ldots \ldots$ & 44 & 4,22 & Anemia carencial ... ... & 40 & 90,90 \\
\hline $\mathrm{V}$ & Mentales y psiconeurosis $\ldots \ldots \ldots$ & 15 & 1,43 & Histeria $\ldots \ldots \ldots$ & 5 & 33,33 \\
\hline VI & Nerviosas y órganos de los sentidos. & 33 & 3,16 & Epilepsia $\ldots \ldots \ldots$ & 13 & 39,39 \\
\hline VII & $\begin{array}{lllllllll}\text { Circulatorias } \ldots & \ldots & \ldots & \ldots & \ldots & \ldots\end{array}$ & 61 & 5,85 & Tromboflebitis $\ldots \ldots \ldots$ & 15 & 24,59 \\
\hline VIII & Respiratorias $\ldots \ldots \ldots$ & 150 & 14,39 & Bronquitis $\ldots \ldots \ldots$ & 65 & 43,33 \\
\hline IX & $\begin{array}{lllllll}\text { Digestivas } \ldots & \ldots & \ldots & \ldots & \ldots & \ldots\end{array}$ & 168 & 16,12 & Apendicitis $\ldots \ldots \ldots \ldots$ & 17 & 10,11 \\
\hline $\mathrm{X}$ & Génito-urinarias $\ldots \ldots \ldots$ & 285 & 27,35 & Metropatías hemorrágicas . & 104 & 36,49 \\
\hline XI & $\begin{array}{l}\text { Parto y puerperio (movimiento asisten- } \\
\text { cial) }\end{array}$ & & & & & \\
\hline XII & $\begin{array}{lllllll}\text { Cutáneas } & \ldots & \ldots & \ldots & \ldots & \ldots & \ldots\end{array}$ & 30 & 2,86 & 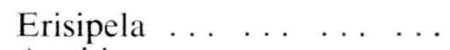 & 4 & 13,33 \\
\hline XIII & Huesos, órganos de locomoción . . . . . . & 7 & 0,67 & Artritis $\ldots \ldots \ldots \ldots$ & 3 & 42,85 \\
\hline XIV & 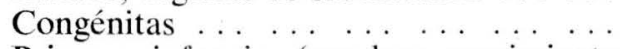 & 4 & 0,38 & Cardiopatías $\ldots \ldots \ldots$ & 2 & 50,00 \\
\hline $\mathrm{XV}$ & $\begin{array}{l}\text { Primera infancia (cuadros movimiento } \\
\text { infantil) }\end{array}$ & & & & & \\
\hline \multirow{2}{*}{$\begin{array}{r}\text { XVI } \\
\text { XVII }\end{array}$} & Síntomas, síndromes $\ldots \ldots \ldots$ & 2 & 0,19 & Uremia $\ldots \ldots$ & 1 & 50,00 \\
\hline & 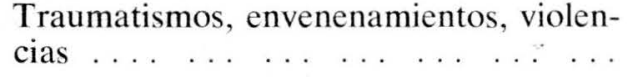 & 22 & 2,11 & Envenenamientos $\ldots \ldots$ & 3 & 13,63 \\
\hline
\end{tabular}




\section{CUADRO C}

INSTITUTO MATERNO INFANTIL

MORBILIDAD MATERNA (NO OBSTETRICA) EN EL AÑO DE 1962

CLASIFICADA SEGUN LOS GRUPOS ESTADISTICOS DE LA O.M.S.

TOT AL: 1.610

\begin{tabular}{|c|c|c|c|c|c|c|}
\hline \multicolumn{2}{|c|}{ Grupos } & \multirow{2}{*}{$\frac{\text { Total }}{73}$} & \multirow{2}{*}{$\frac{\%}{4,53}$} & Particularmente & \multirow{2}{*}{$\frac{\text { Total }}{7}$} & \multirow{2}{*}{$-\frac{\%}{9,58}$} \\
\hline I & Infecciosas y parasitarias $\ldots \ldots \ldots$ & & & T. B. C. pulmonar ... & & \\
\hline II & Neoplasmas $\ldots \ldots \ldots \ldots$ & 104 & 6,45 & Carcinoma cérvix ... ... & 17 & 16,34 \\
\hline III & Alérgicas, endocrinas, nutricionales ... & 43 & 2,67 & Asma $\ldots \ldots \ldots \ldots$ & 15 & 34,88 \\
\hline IV & Sangre y hematopoyéticos $\ldots \ldots \ldots$ & 53 & 3,29 & Anemia carencial $\ldots \ldots$ & 49 & 92,45 \\
\hline $\mathrm{V}$ & Mentales y psiconeurosis $\ldots \ldots \ldots$ & 11 & 0,68 & Histeria $\ldots \ldots \ldots$ & 4 & 36,36 \\
\hline VI & Nerviosas y órganos de los sentidos ... & 31 & 1,92 & Epilepsia . . . . . . . . & 12 & 38,70 \\
\hline VII & Circulatorias $\ldots \ldots \ldots$ & 59 & 3,66 & Tromboflebitis $\ldots \ldots \ldots$ & 15 & 25,42 \\
\hline VIII & Respiratorias . . . . . . . . . . . . . & 138 & 8,57 & Bronconeumonías & 27 & 19,56 \\
\hline IX & Digestivas $\ldots \ldots \ldots \ldots$ & 170 & 10,55 & Enteritis $\ldots \ldots \ldots$ & 12 & 7,05 \\
\hline $\mathrm{X}$ & 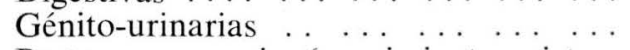 & 842 & 46,08 & Anexitis $\ldots \ldots \ldots$ & 116 & 13,77 \\
\hline XI & $\begin{array}{l}\text { Parto y puerperio (movimiento asisten- } \\
\text { cial) }\end{array}$ & & & & & (1) \\
\hline XII & Cutáneas $\ldots \ldots \ldots \ldots \ldots$ & 20 & 1,24 & Piodermitis $\ldots$. . $\ldots . .$. & 20 & 100,00 \\
\hline XIII & Huesos, órganos de locomoción . . . . . & 6 & 0,37 & Artritis $\ldots \ldots \ldots$ & 5 & 83,33 \\
\hline XIV & 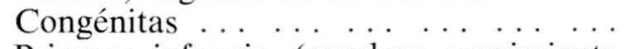 & 11 & 0,68 & Luxación cadera ....... . & 3 & 27,27 \\
\hline XV & $\begin{array}{l}\text { Primera infancia (cuadros movimiento } \\
\text { infantil) }\end{array}$ & & & & & \\
\hline XVI & Síntomas, síndromes $\ldots \ldots \ldots$ & 7 & 0,43 & Uremia $\ldots \ldots$ & 2 & 28,57 \\
\hline XVII & $\begin{array}{l}\text { Traumatismos, envenenamientos, violen- } \\
\text { cias } \ldots \ldots \ldots \\
\ldots\end{array}$ & 42 & 2,60 & Envenenamiento & 1 & 2,38 \\
\hline
\end{tabular}




\section{CUADRO D}

INSTITUTO MATERNO INFANTIL

MORBILIDAD MATERNA (NO OBSTETRICA) EN EL AÑO DE 1963

CLASIFICADA SEGUN LOS GRUPOS ESTADISTICOS DE LA O.M.S.

TOTAL 1.543

\begin{tabular}{|c|c|c|c|c|c|c|c|}
\hline \multicolumn{2}{|c|}{ Grupos } & \multirow{2}{*}{$\frac{\text { Total }}{107}$} & \multirow{2}{*}{$\frac{\%}{6,934}$} & \multicolumn{2}{|l|}{ Particularmente } & \multirow{2}{*}{$\frac{\text { Total }}{9}$} & \multirow{2}{*}{$\frac{\%}{8,41}$} \\
\hline I & Infecciosas y parasitarias $\ldots \ldots \ldots$ & & & T. B. C. pulmonar ... . & & & \\
\hline II & 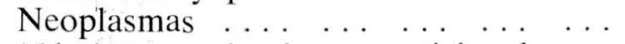 & 104 & 6,740 & Carcinoma cérvix ... & . . & 15 & 14,42 \\
\hline III & Alérgicas, endocrinas, nutricionales .. & 42 & 2,721 & Asma $\ldots \ldots \ldots \ldots$. & . . & 22 & 52.38 \\
\hline IV & Sangre y hematopoyéticos $\ldots \ldots \ldots$ & 33 & 2,141 & Anemia carencial .... & . . & 16 & 48,48 \\
\hline $\mathrm{V}$ & Mentales y psiconeurosis ... . . . . . & 13 & 0,842 & Oligofrenia $\ldots \ldots \ldots$. & . . & 6 & 46,15 \\
\hline VI & Nerviosas y órganos de los sentidos. & 20 & 1,296 & Epilepsia ... . . . . . & . . & 0 & 0 \\
\hline VII & $\begin{array}{llllllll}\text { Circulatorias } & \ldots & \ldots & \ldots & \ldots & \ldots & \ldots\end{array}$ & 159 & 10,304 & Tromboflebitis ... ... & $\ldots$ & 34 & 21,38 \\
\hline VIII & $\begin{array}{lllllll}\text { Respiratorias } & \ldots & \ldots & \ldots & \ldots & \ldots\end{array}$ & 65 & 4,212 & Bronconeumonías . . . & $\ldots$ & 33 & 50,76 \\
\hline IX & Digestivas $\ldots \ldots \ldots$ & 262 & 16,981 & Entëritis $\ldots \ldots$ & $\ldots$ & 12 & 4,58 \\
\hline $\mathrm{X}$ & 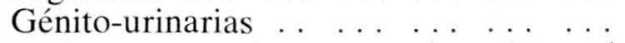 & 629 & 40,764 & Anexitis $\ldots \ldots \ldots$ & $\ldots$ & 155 & 24,64 \\
\hline XI & $\begin{array}{l}\text { Embarazo, parto, puerperio. (Ver el } \\
\text { cuadro sinóptico del movimiento asis- } \\
\text { tencial) }\end{array}$ & & & $\lim _{\rightarrow \infty}$ & & & \\
\hline XII & 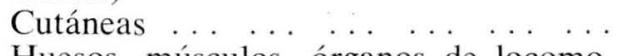 & 15 & 0,972 & Piodermitis $\ldots \ldots \ldots$ & . & 1 & 6,66 \\
\hline XIII & $\begin{array}{l}\text { Huesos, músculos, órganos de locomo- } \\
\text { ción } \ldots \\
\ldots\end{array}$ & 11 & 0,712 & Artritis ... & & 6 & 54,54 \\
\hline XIV & $\begin{array}{llllllll}\text { Congénitas } & \ldots & \ldots & \ldots & \ldots & \ldots & \ldots\end{array}$ & 10 & $=0,651$ & Luxación cadera .... & . . & 1 & 10,00 \\
\hline XVI & Síntomas, síndromes $\ldots \ldots \ldots$ & 16 & 1,036 & Uremia $\ldots . . . .$. & . . & 2 & 12,50 \\
\hline XVII & Traumatismos, violencias $\ldots \ldots$ & 57 & 3,694 & Envenenamiento fósforo & . & 4 & 7,01 \\
\hline
\end{tabular}


CUADRO E

INSTITUTO MATERNO INFANTIL

MORBILIDAD MATERNA (NO OBSTETRICA) EN EL AÑO DE 1964

CLASIFICADA SEGUN LOS GRUPOS ESTADISTICOS DE LA O.M.S.

Total 1.778

\begin{tabular}{|c|c|c|c|c|c|c|}
\hline \multicolumn{2}{|c|}{ Grupos } & \multirow{2}{*}{$\frac{\text { Total }}{196}$} & \multirow{2}{*}{$\frac{\%}{11,02}$} & Particularmente & \multirow{2}{*}{$\frac{\text { Total }}{11}$} & \multirow{2}{*}{$\frac{\%}{5,61}$} \\
\hline I & Infecciones y parasitarias (lúes 105). & & & T. B. C. pulmonar ... . . . & & \\
\hline II & 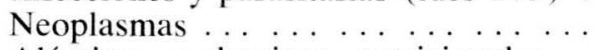 & 107 & 6,02 & Carcinoma cérvix $\ldots \ldots$ & 12 & 11,21 \\
\hline III & Alérgicas, endocrinas, nutricionales .. & 57 & 3,21 & Asma $\ldots \ldots \ldots$ & 15 & 26,32 \\
\hline IV & Sangre y hematopoyéticos $\ldots \ldots \ldots$ & 20 & 1,12 & Anemia aguda $\ldots \ldots \ldots$ & 12 & 60,00 \\
\hline V & Mentales y psiconeurosis . . . . . . & 10 & 0,56 & Oligofrenia $\ldots \ldots \ldots$ & 2 & 20,00 \\
\hline VI & Nerviosas y órganos de los sentidos .. & 37 & 2,08 & Epilepsia $\ldots \ldots \ldots$ & 16 & 43,24 \\
\hline VII & Sistema circulatorio $\ldots \ldots \ldots$ & 166 & 9,35 & Tromboflebitis . . . . . & 35 & 21,08 \\
\hline VIII & Sistema respiratorio $\ldots \ldots \ldots \ldots$ & 83 & 4,67 & Neumonía-broncon. . . . . . . & 44 & 53,01 \\
\hline IX & $\begin{array}{llllll}\text { Digestivas } & \ldots & \ldots & \ldots & \ldots & \ldots\end{array}$ & 160 & 9,00 & Enteritis $\ldots \ldots \ldots$ & 5 & 3,13 \\
\hline $\mathrm{X}$ & Génito urinarias $\ldots \ldots \ldots \ldots$ & 853 & 47,98 & Anexitis $\ldots \ldots \ldots$ & 189 & 22,16 \\
\hline XI & $\begin{array}{l}\text { Embarazo, parto, puerperio. (Ver cua- } \\
\text { dro sinóptico (Y) del movimiento } \\
\text { asistencial) }\end{array}$ & & & & & \\
\hline XII & $\begin{array}{lllllll}\text { Cutáneas } & \ldots & \ldots & \ldots & \ldots & \ldots & \ldots\end{array}$ & 20 & 1.12 & Piodermitis $\ldots . \ldots \ldots$ & 8 & 40,00 \\
\hline XIII & Aparato locomotor $\ldots \ldots \ldots$ & 10 & 0,56 & $\begin{array}{lllll}\text { Artritis } & \ldots & \ldots & \ldots & \ldots\end{array}$ & 3 & 30,00 \\
\hline XIV & Congénitas $\ldots \ldots \ldots \ldots$ & 9 & 0,51 & Cardiopatía $\ldots \ldots \ldots$ & 1 & 11,11 \\
\hline XV & $\begin{array}{l}\text { Primera infancia. (Ver cuadro movi } \\
\text { miento infantil) }\end{array}$ & & & & & \\
\hline XVI & Síntomas, síndromes $\ldots \ldots \ldots$ & 16 & 0,89 & Retención de orina ... . . . & 4 & 25,00 \\
\hline XVII & $\begin{array}{l}\text { Traumatismos, violencias, envenena- } \\
\text { miento } \ldots \ldots \ldots \ldots \ldots \ldots \ldots\end{array}$ & 34 & 1.91 & Envenenamiento con fósforo & 3 & 8,82 \\
\hline
\end{tabular}




\author{
CUADRO F \\ MORBILIDAD DEL RECIEN NACIDO \\ EN EL INSTITUTO MATERNO INFANTIL \\ Años 1960 y 1961
}

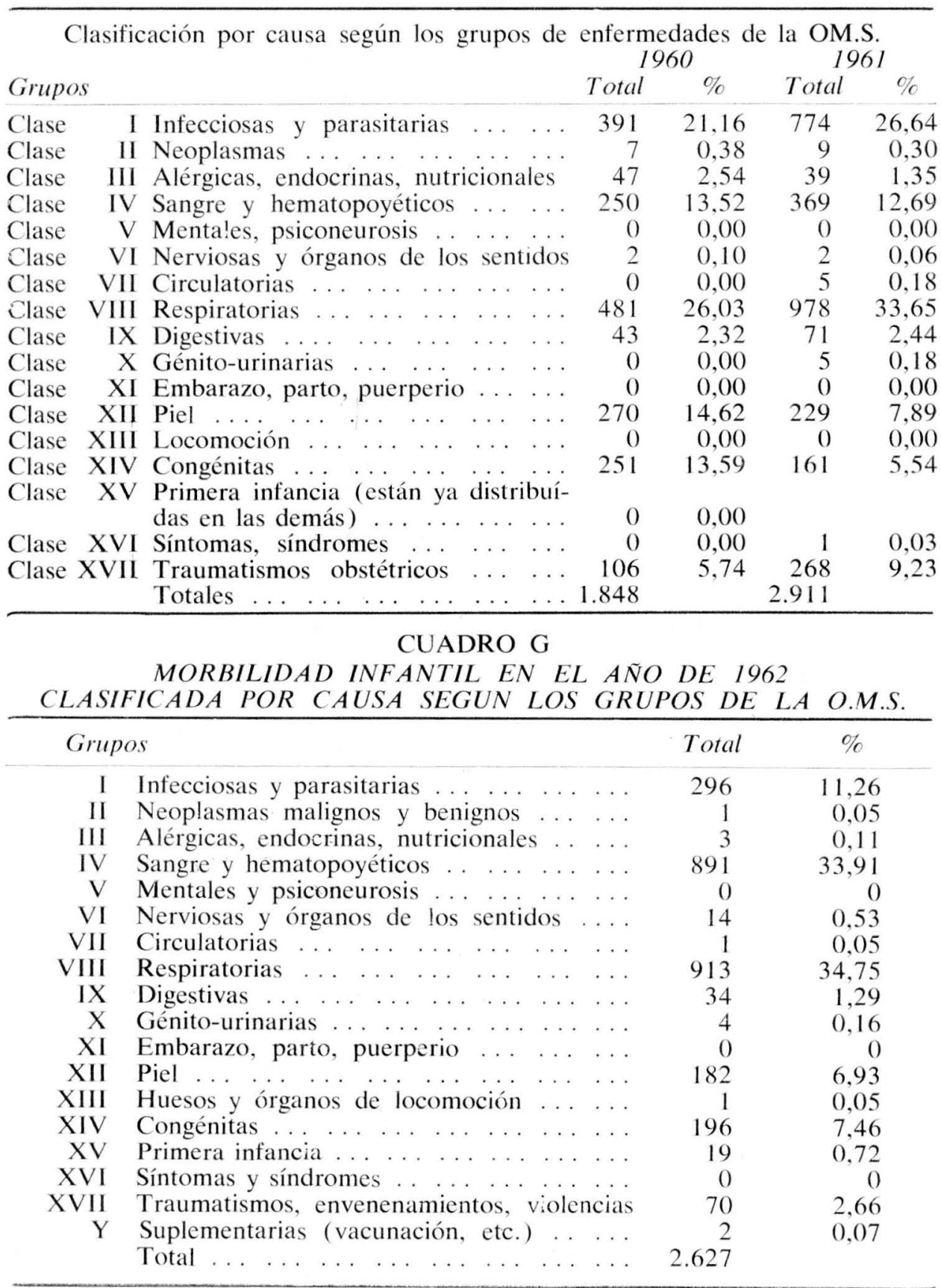




\section{CUADRO $\mathrm{H}$}

MORTALIDAD INFANTIL EN EL AÑO DE 1963

CLASIFICADA SEGUN LOS GRUPOS PATOLOGICOS ESTADISTICOS DE LA O.M.S.

\begin{tabular}{|c|c|c|c|}
\hline \multicolumn{2}{|c|}{ Grupos } & \multirow{2}{*}{\begin{tabular}{|l} 
Total \\
396
\end{tabular}} & \multirow{2}{*}{$\begin{array}{r}\% \\
18,92 \\
\end{array}$} \\
\hline I & Infecciones y parasitarias $\ldots \ldots \ldots$ & & \\
\hline II & Neoplasmas $\ldots \begin{array}{llllll} & \ldots & \ldots & \ldots & \ldots & \ldots\end{array}$ & 4 & 0,19 \\
\hline III & Alérgicas, endocrinas, nutricionales $\ldots \ldots$ & 31 & 1,48 \\
\hline IV & Sangre y hematopoyéticos $\ldots \ldots \ldots$ & 451 & 21,54 \\
\hline V & Mentales y psiconeurosis $\ldots \ldots \ldots$ & 0 & 0 \\
\hline VI & Nerviosas y órganos de los sentidos ... & 5 & 0,24 \\
\hline VII & Circulatorias $\ldots \ldots \ldots$ & 7 & 0,34 \\
\hline VIII & Respiratorias $\ldots \ldots \ldots$ & 490 & 23,40 \\
\hline IX & 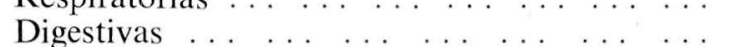 & 157 & $\begin{array}{r}2,40 \\
7,49\end{array}$ \\
\hline $\mathrm{X}$ & $\begin{array}{lllllll}\text { Génito-urinarias } & \ldots & \ldots & \ldots & \ldots & \ldots & \ldots\end{array}$ & 8 & 0,38 \\
\hline XI & Embarazo, parto, puerperio $\ldots \ldots \ldots$ & 0 & 0 \\
\hline XII & Cutáneas $\ldots \ldots \ldots$ & 141 & 6,73 \\
\hline XIII & Huesos, músculos, órganos de locomoción . . & 0 & () \\
\hline XIV & 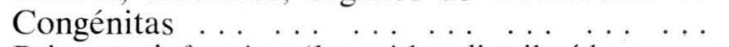 & 328 & 15,67 \\
\hline XV & $\begin{array}{l}\text { Primera infancia (ha sido distribuído entre } \\
\text { los otros grupos). }\end{array}$ & & \\
\hline XVI & Síntomas, síndromes $\ldots \ldots \ldots$ & 0 & 0 \\
\hline \multirow[t]{2}{*}{ XVII } & Traumas obstétricos, violencias $\ldots \ldots \ldots$ & 76 & 3,62 \\
\hline & $\begin{array}{lllllllll}\text { Total } & \ldots & \ldots & \ldots & \ldots & \ldots & \ldots & \ldots & \ldots\end{array}$ & 2.094 & \\
\hline
\end{tabular}

\section{CUADRO I}

MORTALIDAD INFANTIL EN EL AÑO DE 1960 CLASIFICADA SEGUN LOS GRUPOS PATOLOGICOS ESTADISTICOS DE LA O.M.S.

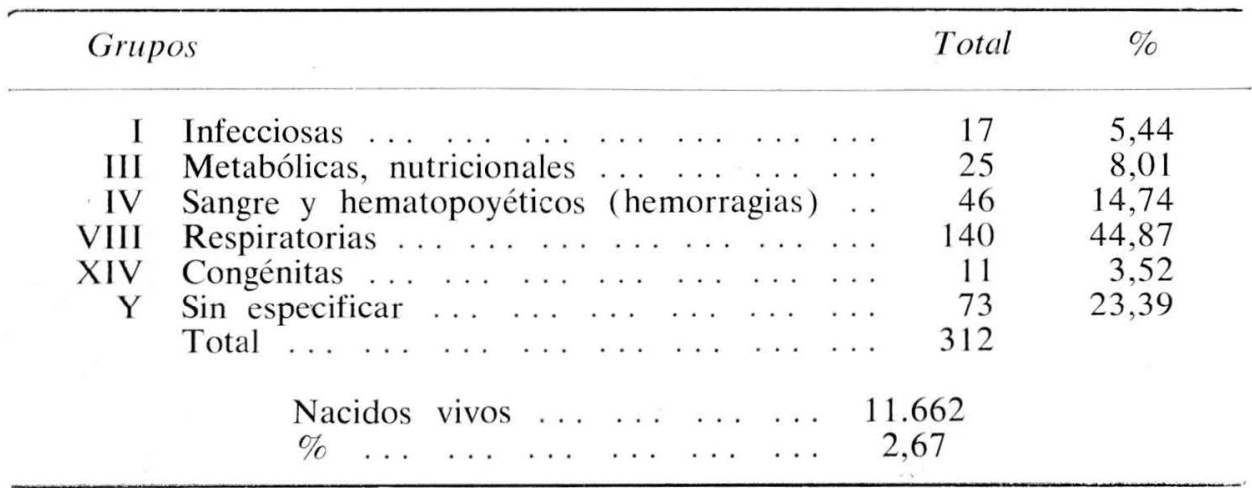




\section{CUADRO J \\ MORTALIDAD INFANTIL EN EL AÑO DE 1961 \\ CLASIFICADA SEGUN LOS GRUPOS PATOLOGICOS ESTADISTICOS DE LA O.M.S.}

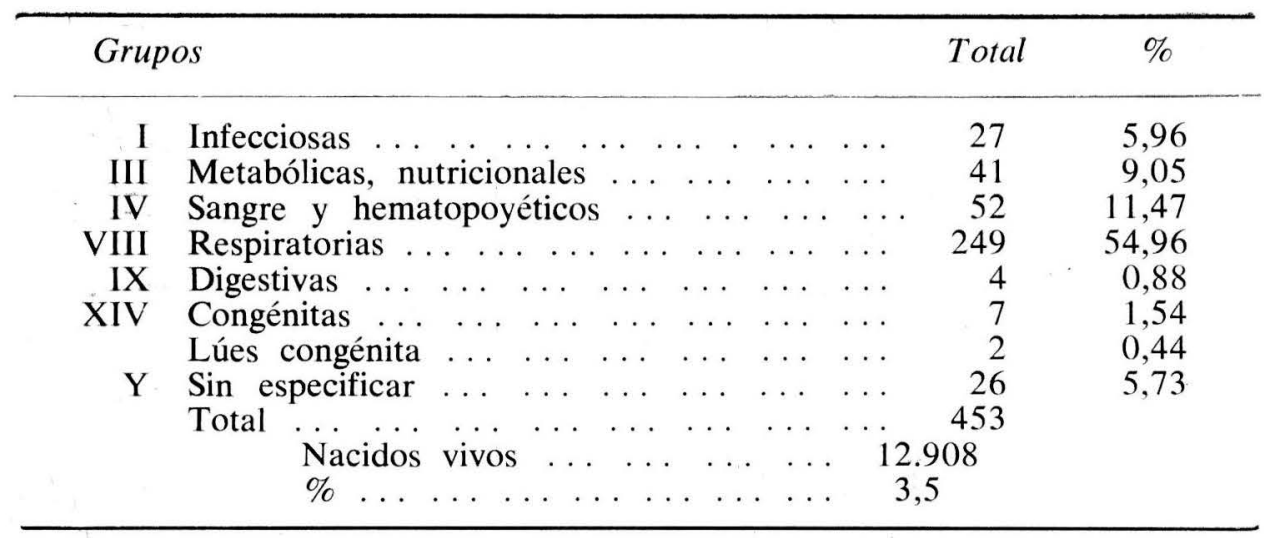

\section{CUADRO K}

MORTALIDAD INFANTIL EN EL AÑO DE 1962

CLASIFICADA SEGUN LOS GRUPOS PATOLOGICOS ESTADISTICOS DE LA O.M.S

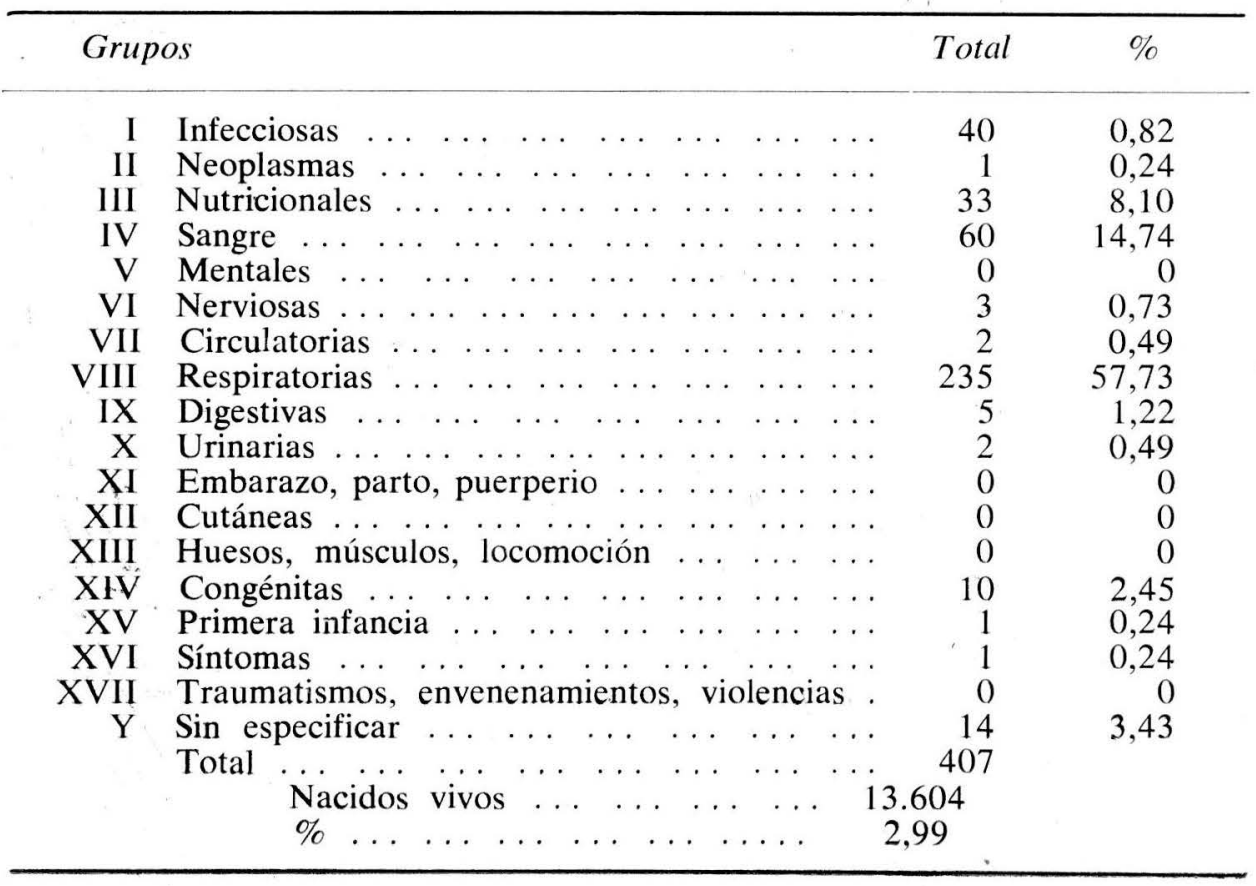


CUADRO L

DEFUNCIONES INFANTILES NEONATALES EN EL AÑO DE 1963

CLASIFICADAS POR CAUSAS SEGUN LOS GRUPOS ESTADISTICOS DE LA O.M.S.

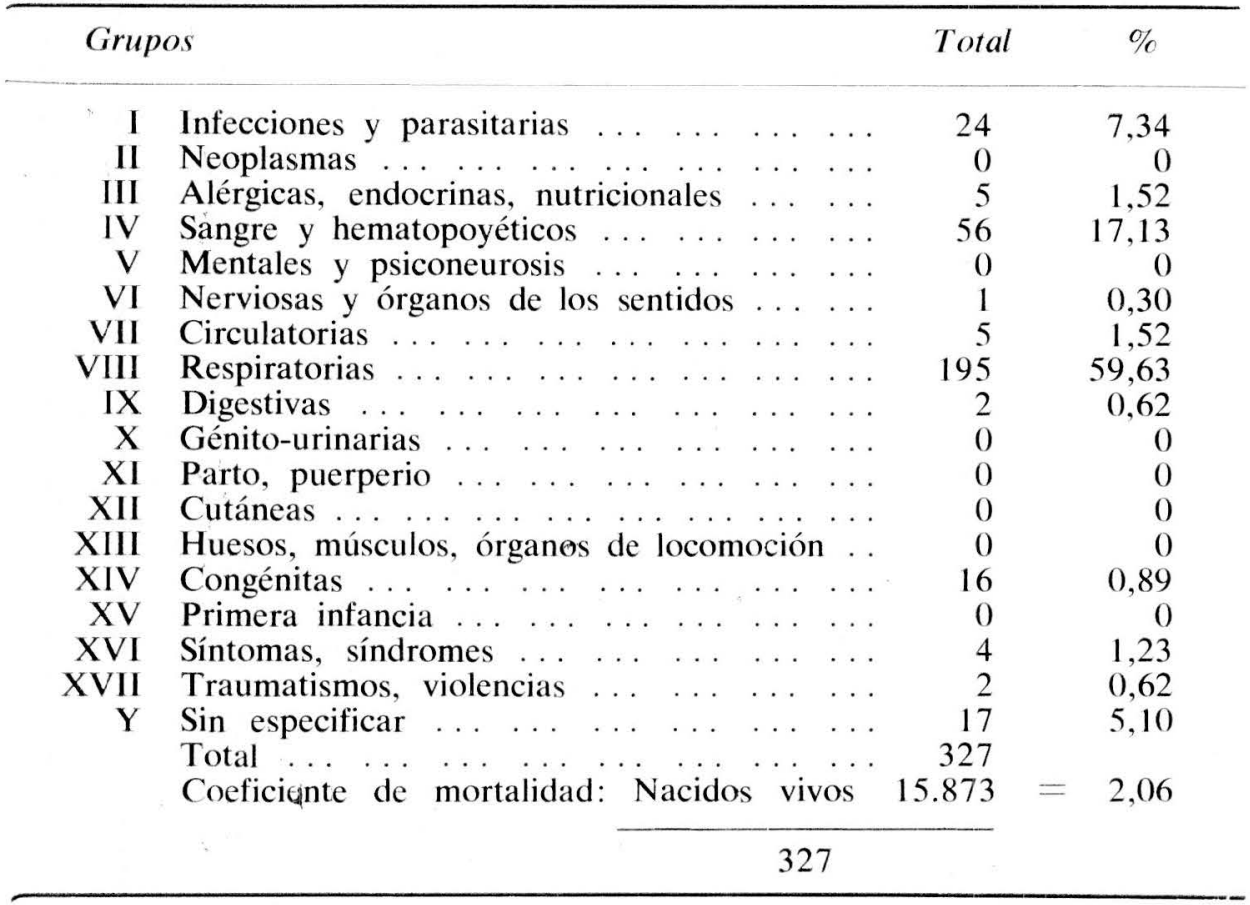

Nota:

Las defunciones por inmadurez dan un total de $\quad 44=\% \quad 0,27$

Porcentaje sobre nacidos vivos $=0,27 \%$

"SEGUROS MEDICOS VOLUNTARIOS, S. A.",

Es la única compañía del país especializada en e! ramo de Salud.

Solicite informes: Apartado Aéreo Nơ 11777 - Bogotá, D. E. 
DEFUNCIONES INFANTILES NO NEONATALES EN EL AÑO DE 1963 CLASIFICADAS SEGUN LOS GRUPOS ESTADISTICOS DE LA O.M.S.

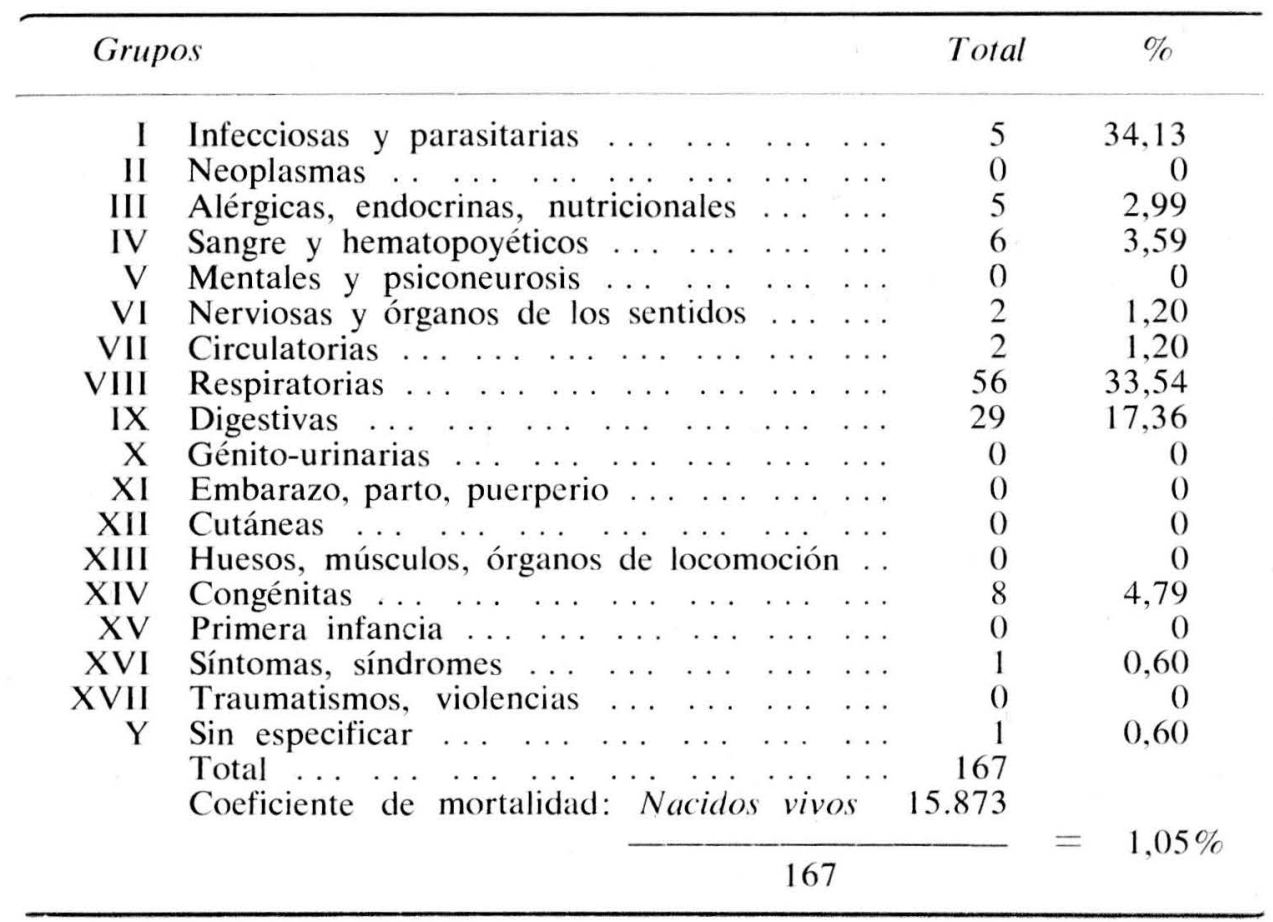




\section{CUADRO O}

INSTITUTO MATERNO INFANTIL

MORBILIDAD INFANTIL EN EL AÑO 1964

\section{CLASIFICADA SEGUN LOS GRUPOS ESTADISTICOS DE LA O.M.S.}

Total 4.163

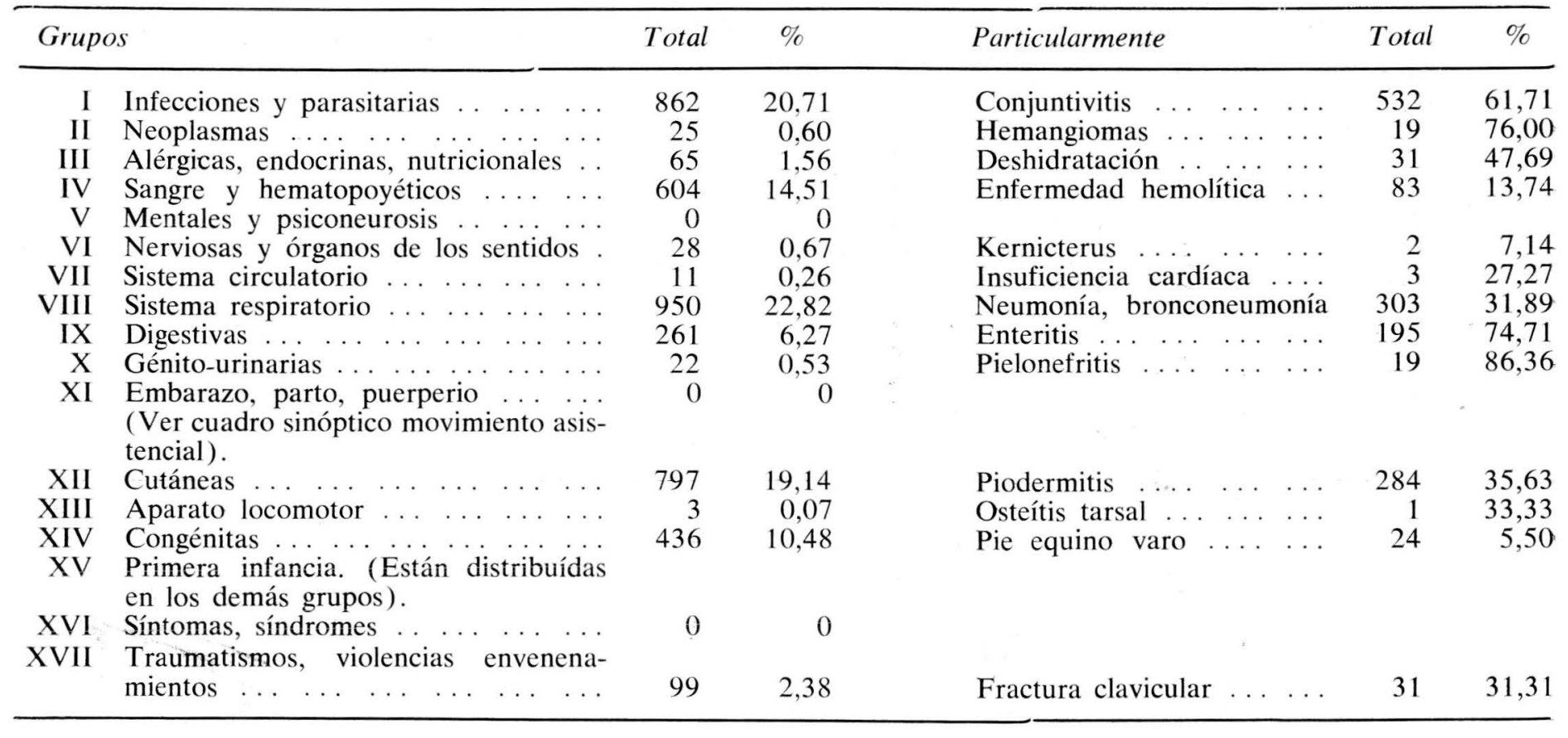


CUADRO P

DEFUNCIONES INFANTILES NEONATALES EN 1964, CLASIFICADOS SEGUN LOS GRUPOS PATOLOGICOS ESTADISTICOS DE LA O.M.S. COMPARACION CON EL AÑO 1963

\begin{tabular}{|c|c|c|c|c|c|c|c|}
\hline Grupo & Descripción & & & 1963 & 1964 & & $\%$ \\
\hline I & Infecciones y parasitarias .. & $\ldots$ & $\ldots$ & 24 & 13 & - & 45,83 \\
\hline II & Neoplasmas $\ldots \ldots \ldots \ldots$ & $\ldots$ & . & 0 & 0 & & - \\
\hline III & Alérgicas, endocrinas, nutricionales & $\ldots$ & $\ldots$ & 5 & 10 & + & 100,00 \\
\hline IV & Sangre y hematopoyéticos $\ldots \ldots$ & $\ldots$ & $\ldots$ & 56 & 36 & - & 35,71 \\
\hline V & Mentales y psiconeurosis $\ldots \ldots \ldots$ & $\ldots$. & $\ldots$ & 0 & 0 & & - \\
\hline VI & Nerviosas y órganos de los sentidos & $\ldots$ & $\ldots$ & 1 & 2 & + & 100,00 \\
\hline VII & Circulatorias $\ldots \ldots \ldots$ & $\ldots$ & $\ldots$ & 5 & 7 & + & 40,00 \\
\hline VIII & Respiratorias $\ldots \ldots \ldots$ & $\ldots$ & $\ldots$ & 195 & 191 & - & 2,05 \\
\hline IX & $\begin{array}{lllllll}\text { Digestivas } & \ldots & \ldots & \ldots & \ldots & \ldots\end{array}$ & $\ldots$ & . . & 2 & 2 & & - \\
\hline $\mathrm{X}$ & Urinarias $\ldots \ldots \ldots$ & $\ldots$ & $\ldots$ & 0 & 1 & + & 100,00 \\
\hline XI & Parto, puerperio $\ldots \ldots \ldots$ & $\ldots$ & $\ldots$ & 0 & 0 & & - \\
\hline XII & 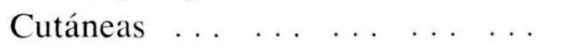 & $\ldots$ & . . & 0 & 0 & & - \\
\hline XIII & Huesos, músculos y locomoción & $\ldots$ & . & 0 & 0 & & - \\
\hline XIV & Congénitas $\ldots \begin{array}{lllll} & \ldots & \ldots & \ldots & \ldots\end{array}$ & $\ldots$ & $\ldots$ & 16 & 4 & - & 75,00 \\
\hline XV & Primera infancia $\ldots \ldots \ldots$ & $\ldots$ & $\ldots$ & - & - & & - \\
\hline XVI & Síntomas, síndromes $\ldots \ldots \ldots$ & $\ldots$ & $\ldots$ & 4 & 2 & - & 50,00 \\
\hline XVII & Traumatismos, violencias ... & $\ldots$ & . & 2 & 1 & - & 50,00 \\
\hline \multirow[t]{2}{*}{$\mathrm{Y}$} & sin especificar $\ldots \ldots \ldots$ & $\ldots$ & . & 17 & 8 & - & 52,94 \\
\hline & Totales ... & $\ldots$ & $\ldots$ & 327 & 277 & & \\
\hline
\end{tabular}

1963 Coeficiente de mortalidad Nacidos vivos. $\ldots . \ldots . . .15 .873$

Defunciones . . . . . . . . . $\frac{1}{327}=2,06 \%$

1964 Nacidos vivos . . . . . . . 17.392

Defunciones ... ........ . 54543

1963 Defunciones por inmadurez . . 44

Nacidos vivos ... . . . . . 15.873

1964 Defunciones por inmadurez . . 60

Nacidos vivos $\frac{60}{17.392}=0,34 \%$ 
CUADROQ

MORTALIDAD INFANTIL (NO NEONATAL) EN EL AÑO 1964

CLASIFICADO SEGUN LOS GRUPOS PATOLOGICOS

EST ADISTICOS DE LA O.M.S

(Comparación con el año 1963)

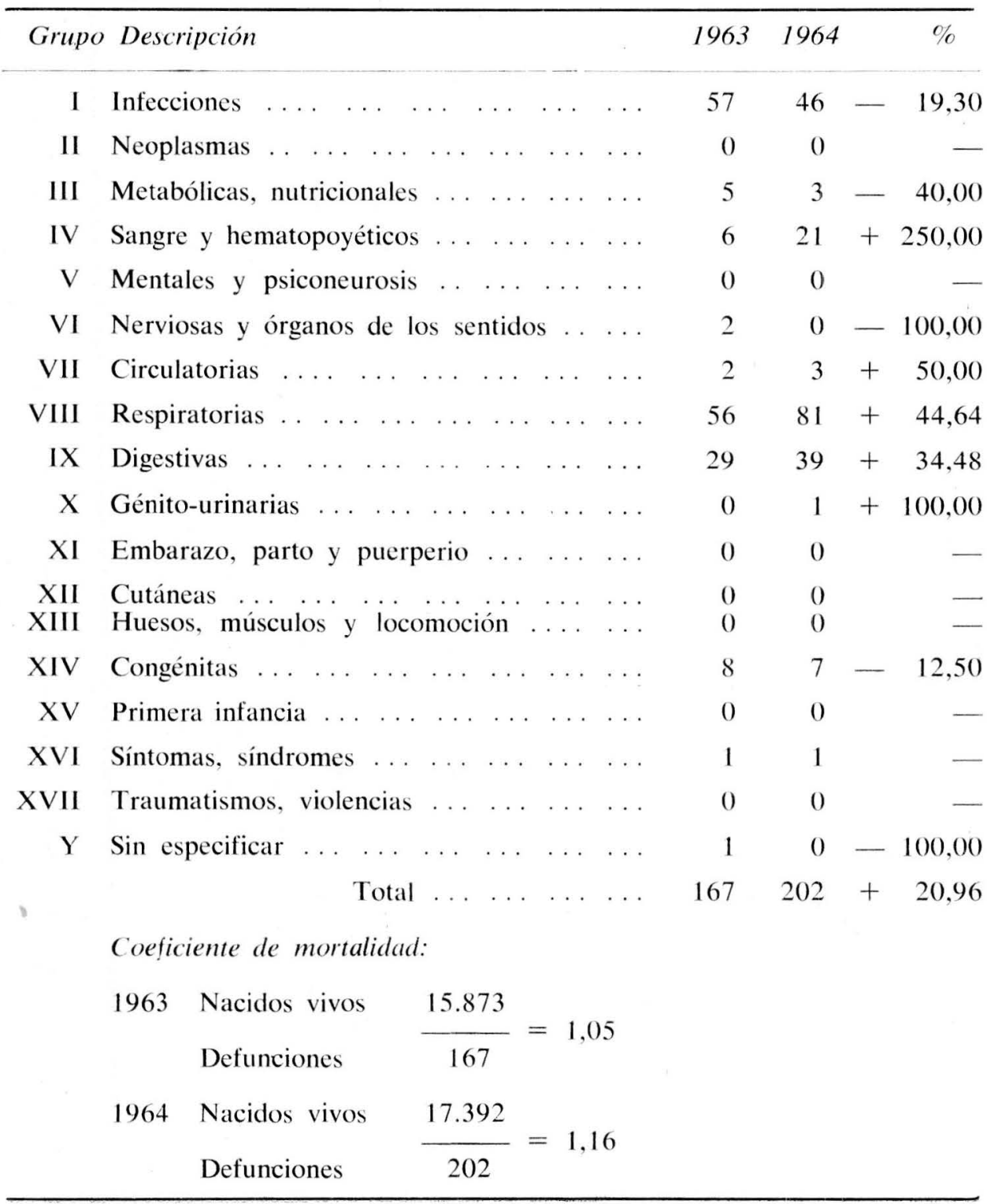


DEFUNCIONES MATERNAS SEGUN INDICE. Código "Standard" 7x0-y01 $A \tilde{N} O \quad D E \quad 1961$

\begin{tabular}{|c|c|c|}
\hline$N^{o}$ Historia & Edad & Censo principal de la defunción según historia \\
\hline 78.285 & 19 & Intoxicación con fósforo. \\
\hline 78.429 & 19 & Intoxicación con fósforo. \\
\hline 78.420 & 24 & Aborto séptico. \\
\hline 78.412 & $?$ & Putrefacción fetal, insuficiencia renal. \\
\hline 79.091 & 40 & Ulcera sangrante, anemia aguda. \\
\hline 78.920 & 41 & Embolia pulmonar. \\
\hline 80.188 & 28 & Eclampsia, hemorragia cerebral. \\
\hline 64.236 & 39 & Retención de placenta. \\
\hline 80.626 & $?$ & Hemorragia cerebral. \\
\hline 80.694 & 35 & Hemorragia cerebral. \\
\hline 18.115 & 29 & Aborto séptico, pelviperitonitis. \\
\hline 81.112 & 17 & "Shock" hemorrágico, paro cardíaco. \\
\hline 54.876 & 35 & Aborto séptico, sepsis a estafilococo. \\
\hline 11.597 & 42 & Placenta previa, "shock" hemorrágico. \\
\hline 81.354 & 25 & Bronconeumonía. \\
\hline 8.952 & 19 & Hemorragia cerebral. \\
\hline$?$ & $?$ & Intoxicación por fósforo. \\
\hline 84.126 & 28 & Aborto séptico. \\
\hline 67.557 & 22 & Hemorragia cerebral. \\
\hline 84.651 & 21 & Hemorragia cerebral. \\
\hline 72.602 & 34 & Sin determinar, \\
\hline 14.932 & $?$ & Sin determinar. \\
\hline 70.137 & $?$ & Toxemia. \\
\hline 86.286 & 20 & Meningitis T.B.C. \\
\hline 86.738 & 19 & Mola hidatiforme. \\
\hline 86.707 & 26 & Aborto, pelviperitonitis. \\
\hline 86.948 & 45 & Aborto, edema agudo pulmonar. \\
\hline 61.916 & 20 & Septicemia. \\
\hline 87.635 & 25 & Aborto T.B.C. pulmonar. \\
\hline 87.619 & 33 & Oclusión intestinal, peritonitis. \\
\hline 87.868 & 30 & Perforación uterina, pelviperitonitis. \\
\hline 56.432 & 30 & Aborto provocado. \\
\hline 88.158 & $?$ & Sin determinar. \\
\hline 38.925 & 30 & Aborto provocado. \\
\hline 88.924 & 28 & Bronconeumonía. \\
\hline 89.176 & $?$ & Aborto séptico. \\
\hline 89.520 & 36 & Desprendimiento de la placenta. \\
\hline
\end{tabular}

Total $=37$ 
DEFUNCIONES MATERNAS SEGUN INDICE. Código "Standard" 7x0-y0I AÑO DE 1961

COEFICIENTE GENERAL: Anual

Total de defunciones 37

Total de hospitalizadas y salidas o altas 19.987

\section{COEFICIENTE ESPECIFICO:}

Total de fallecidas entre 0 y 48 horas de hospitalización

Total de salidas o altas. (No se determinó en este informe).

$$
\text { CUADRO R }
$$

DEFUNCIONES MATERNAS SEGUN INDICE. Código "Standard" 7x0-y0I $A \tilde{N} O \quad D E \quad 1960$

\section{No Historia Edad Censo principal de la defunción según historia}

$\begin{array}{lrl}67.687 & 25 & \text { Sepsis generalizada. } \\ 68.009 & 41 & \text { Ruptura uterina. Peritonitis. } \\ 68.205 & 34 & \text { Placenta previa. Anemia aguda. } \\ 44.002 & 23 & \text { Salpingoforectomía. Peritonitis. } \\ 68.002 & 24 & \text { Endometritis. Septicemia puerperal. } \\ 68.457 & 23 & \text { Coma eclámptico. } \\ 68.200 & 27 & \text { Carcinoma cérvix. } \\ 68.481 & 22 & \text { Aborto provocado. Peritonitis. } \\ 42.382 & 35 & \text { Edema pulmonar. Eclampsia. } \\ 68.862 & 38 & \text { Placenta previa. } \\ 68.729 & ? & \text { Eclampsia. } \\ 69.513 & 30 & \text { Pelviperitonitis. } \\ 69.911 & ? & \text { Toxemia. } \\ 69.673 & 37 & \text { Desprendimiento placentario. } \\ 38.831 & 28 & \text { Eclampsia. } \\ 27.281 & 28 & \text { Septicemia. } \\ 71.863 & 37 & \text { Ectópico roto. Anemia aguda. } \\ 72.173 & ? & \text { Peritonitis. } \\ 71.810 & 33 & \text { Septicemia. } \\ 72.399 & ? & \text { Sin dẹterminar. }\end{array}$


DEFUNCIONES MATERNAS SEGUN INDICE. Código "Standard" 7x0-y0I $A \tilde{N} O \quad D E \quad 1960$

\begin{tabular}{rrl}
\hline No Historia & Edad & Censo principal de la defunción según historia \\
\hline 73.215 & 37 & Edema pulmonar. \\
73.663 & 39 & Pancreatitis hemorrágica. \\
73.130 & 42 & Edema pulmonar. \\
72.894 & 17 & Toxemia. Hemorragia cerebral. \\
8.996 & 25 & Cesárea. Edema pulmonar. \\
52.581 & 29 & Peritonitis. \\
74.103 & $?$ & Sin determinar. \\
12.689 & 24 & Aborto, perforación uterina, septicemia. \\
72.536 & $?$ & Sin determinar. \\
73.672 & 27 & Aborto séptico. \\
75.020 & 35 & Insuficiencia cardíaca. \\
41.569 & 31 & Septicemia. \\
75.090 & 36 & "Shock" hemorrágico (parto fuera del Instituto). \\
75.111 & 18 & Eclampsia. \\
75.789 & 30 & Peritonitis (parto fuera del Instituto). \\
74.180 & 35 & Aborto séptico, pelviperitonitis. \\
62.184 & 33 & Cesárea, peritonitis. \\
76.025 & $?$ & Eclampsia. \\
76.242 & $?$ & Toxemia. \\
76.454 & 20 & Edema pulmonar. \\
76.734 & 34 & Hemorragia subaracnoidea. \\
76.759 & $?$ & Ileo paralítico. \\
65.758 & 28 & Intoxicación con fósforo. \\
77.188 & 32 & Sin determinar. \\
76.441 & 29 & Hemorragia cerebral. \\
77.444 & 22 & Intoxicación con fósforo. \\
76.070 & 16 & Embolia arteria pulmonar. \\
77.803 & 18 & Intoxicación con fósforo. \\
77.987 & 37 & Aborto séptico. \\
77.666 & 24 & Ulcera péptica sangrante. \\
21.622 & 38 & Cesárea, "shock", paro cardiaco. \\
75.946 & $?$ & Edema pulmonar. \\
76.966 & $?$ & Embolia pulmonar \\
& &
\end{tabular}

Total $=53$

COEFICIENTE GENERAL: Anual

Total defunciones 53

Total de hospitalizadas y salidas o altas 18.254

COEFICIENTE ESPECIFICO:

Total de fallecidas entre 0 y 48 horas de hospitalización

Total de salidas o altas. (No se determinó en este informe). 
DEFUNCIONES MATERNAS SEGUN INDICE. Código "Standard" 7x0-y0i $A \tilde{N} O \quad D E \quad 1962$

\begin{tabular}{|c|c|c|}
\hline No Historia & Edad & Censo principal de la defunción según historia \\
\hline 26.918 & 35 & Insuficiencia cardíaca. \\
\hline 77.196 & $?$ & Fibrinogenopenia. \\
\hline 63.112 & 35 & Aborto séptico. \\
\hline 27.012 & 37 & Eclampsia, hemorragia cerebral. \\
\hline 40.797 & 25 & Aborto criminal séptico. \\
\hline 91.242 & 31 & Eclampsia, hemorragia cerebral. \\
\hline 33.023 & 25 & Sin determinar. \\
\hline 91.752 & 13 & Glomerulonefritis. bronconeumonía. \\
\hline 92.301 & 50 & Carcinoma cérvix. \\
\hline 92.442 & 28 & Eclampsia, hemorragia cerebral. \\
\hline 93.037 & 33 & Fractura de la base del cráneo. \\
\hline 92.840 & 20 & Meningitis T.B.C. \\
\hline 93.650 & $?$ & Tétanos. \\
\hline 93.452 & $?$ & Anemia. \\
\hline 75.331 & 26 & Aborto séptico. \\
\hline 92.595 & 24 & Peritonitis. \\
\hline 94.103 & 23 & Eclampsia. \\
\hline 4.991 & 27 & Caquexia cancerosa. \\
\hline 95.909 & 38 & Edema pulmonar. \\
\hline 96.105 & 41 & Hemorragia cerebral. \\
\hline 96.654 & 40 & Hemorragia hepática. \\
\hline 22.908 & 37 & Hemorragia del alumbramiento. \\
\hline 96.758 & 54 & Peritonitis, bronconeumonía. \\
\hline 97.870 & 27 & Aborto séptico. \\
\hline 98.272 & 18 & Aborto séptico, peritonitis. \\
\hline 98.483 & 27 & Peritonitis. \\
\hline 93.800 & 30 & Hepatitis. \\
\hline 16.064 & 28 & Ruptura uterina. \\
\hline 82.973 & 27 & Hemorragia cerebral. \\
\hline
\end{tabular}

Total de defunciones 29

Total de hospitalizadas y salidas o altas 21.339.

\section{COEFICIENTE ESPECIFICO:}

Total de fallecidas entre 0 y 48 horas de hospitalización

Total de salidas o altas. (No se determinó en este informe). 
CUADRO T

DEFUNCIONES MATERNAS SEGUN INDICE. Código "Standard" 7x0_y0I $A \tilde{N} O \quad D E \quad 1963$

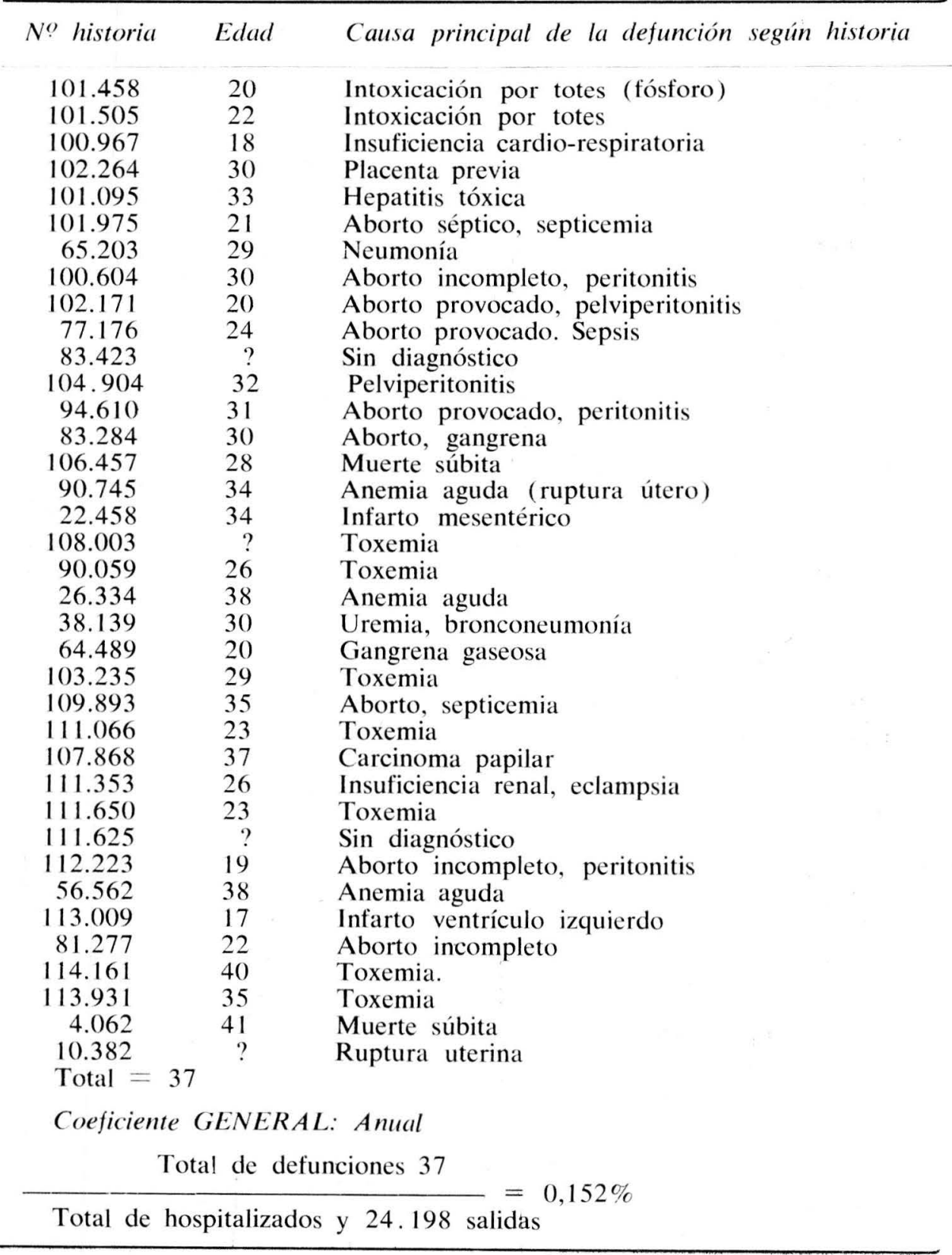


CUADRO U

DEFUNCONES MATERNAS SEGUN INDICE CODIGO STANDARD 7x0-y01 - 1964

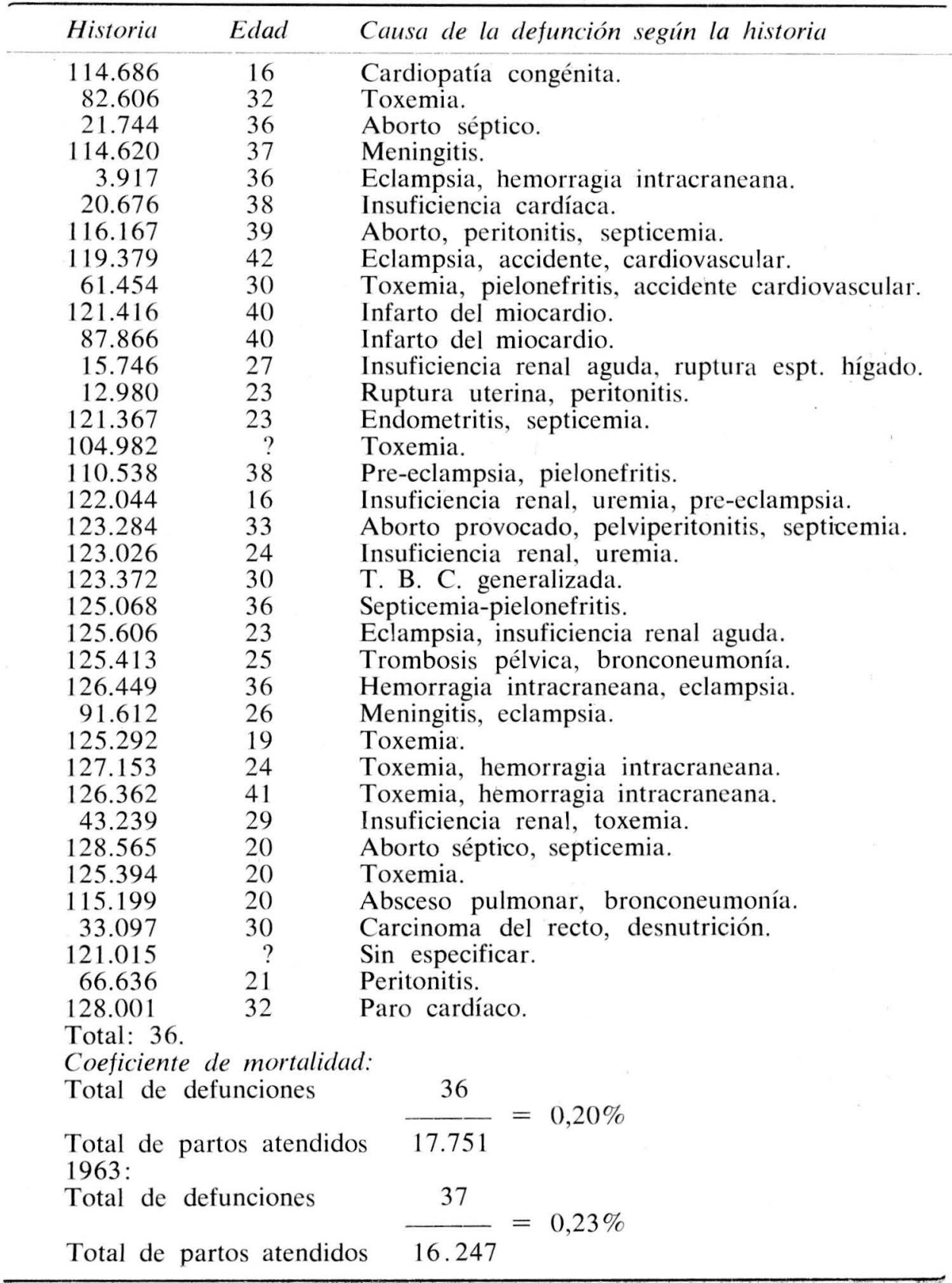


CUADRO X

SINOPSIS DEL MOVIMIENTO CIENTIFICO-ASISTENCIAL DEL INSTITUTO MATERNO INFANTIL "CONCEPCION VILLAVECES DE ACOSTA"

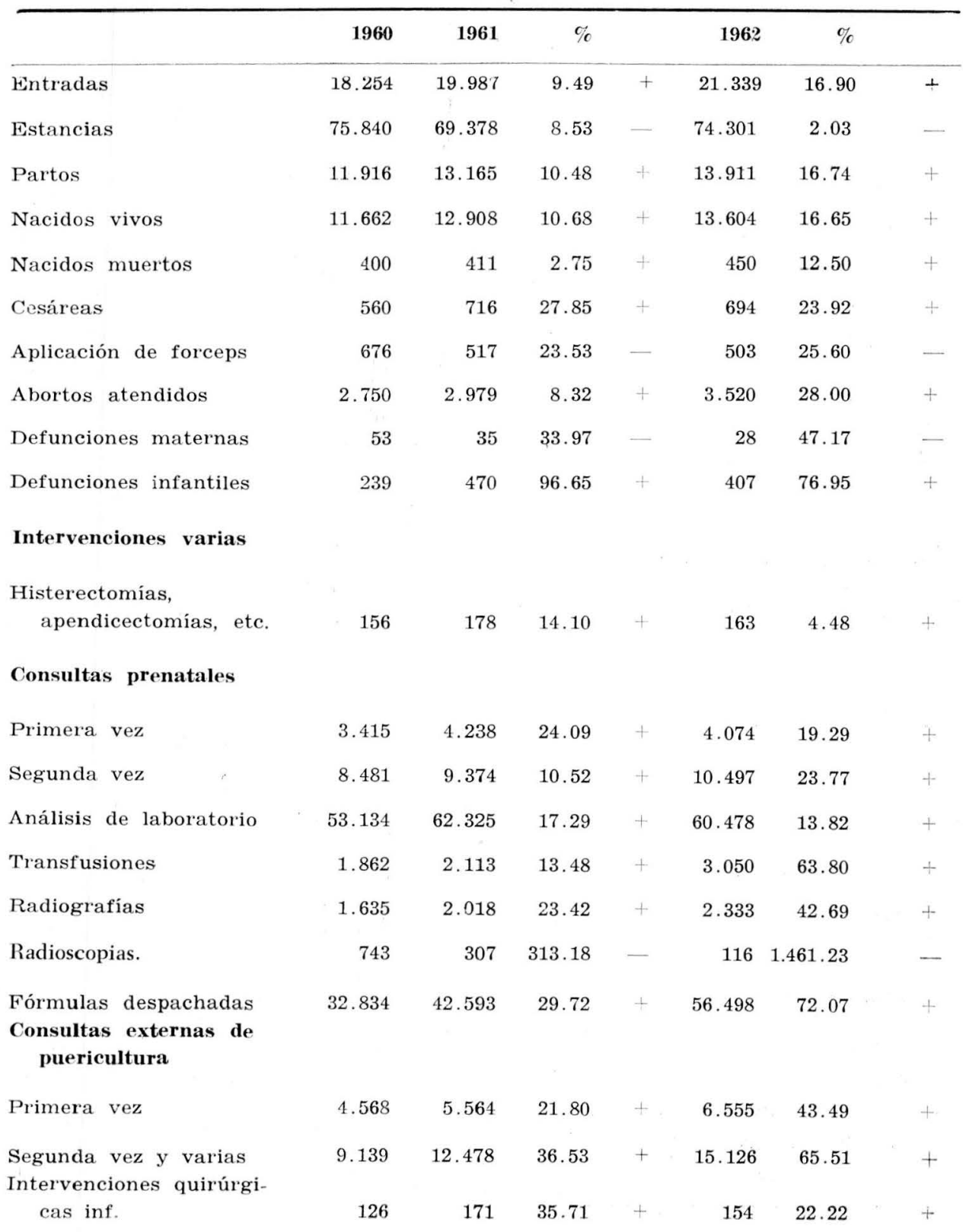


SINOPSIS DEL MOVIMIENTO CIENTIFICO-ASISTENCIAL

DEL INSTITUTO MATERNO INFANTIL "CONCEPCION VILLAVECES DE ACOSTA" - 1963

\begin{tabular}{|c|c|c|c|c|c|}
\hline & & 1962 & 1963 & & $\%$ \\
\hline Entradas $\ldots \ldots \ldots$ & $\ldots \ldots$ & 21.339 & 24,198 & + & 13,29 \\
\hline $\begin{array}{llllllll}\text { Estancias } & \ldots & \ldots & \ldots & \ldots & \ldots & \ldots\end{array}$ & $\ldots \ldots$ & 74.301 & 93.167 & + & 24,39 \\
\hline $\begin{array}{lllllll}\operatorname{Partos} & \ldots & \ldots & \ldots & \ldots & \ldots & \ldots\end{array}$ & $\ldots \ldots$ & 13.911 & 16.247 & + & 16,79 \\
\hline Nacidos vivos $\ldots \ldots \ldots$ & $\ldots \ldots$ & 13.604 & 15.873 & + & 16,67 \\
\hline Nacidos muertos $\ldots \ldots \ldots$ & $\ldots \ldots$ & 450 & 520 & + & 15,55 \\
\hline $\begin{array}{llllllll}\text { Cesáreas } & \ldots & \ldots & \ldots & \ldots & \ldots & \ldots\end{array}$ & $\ldots \ldots$ & 694 & 796 & + & 14,69 \\
\hline Aplicación de forceps $\ldots \ldots \ldots$ & $\ldots \ldots$ & 503 & 1.800 & + & 257,85 \\
\hline Abortos atendidos $\ldots \ldots \ldots$ & $\ldots \ldots$ & 3.520 & 3.852 & + & 9,43 \\
\hline Defunciones maternas ... ... . . & $\ldots \ldots$ & 28 & 37 & + & 32,14 \\
\hline Defunciones infantiles $\ldots \ldots \ldots$ & $\ldots \ldots$ & 407 & 536 & + & 31,69 \\
\hline \multicolumn{6}{|l|}{ Intervenciones varias } \\
\hline Histerectomías, apendicectomías, etc. & $\cdots \cdots$ & 163 & 198 & + & 21,47 \\
\hline \multicolumn{6}{|l|}{ Consultas prenatales } \\
\hline Primera vez $\ldots \begin{array}{lllll} & \ldots & \ldots & \ldots & \ldots\end{array}$ & $\ldots$ & 4.074 & 4.672 & + & 14,67 \\
\hline Segunda vez $\ldots \ldots \ldots$ & $\ldots .$. & 10.497 & 11.497 & + & 11,33 \\
\hline Análisis de laboratorio $\ldots \ldots \ldots$ & $\ldots \ldots$ & 60.478 & 76.657 & + & 26,75 \\
\hline Transfusiones $\ldots \ldots \ldots$ & $\ldots \ldots$ & 3.050 & 2.991 & - & 1.96 \\
\hline Radiografías $\ldots \ldots \ldots$ & $\ldots \ldots$ & 2.333 & 2.101 & - & 9,95 \\
\hline $\begin{array}{lllllll}\text { Radioscopias } & \ldots & \ldots & \ldots & \ldots & \ldots\end{array}$ & $\ldots \ldots$ & 116 & 123 & + & 6,05 \\
\hline \multicolumn{6}{|l|}{ Fórmulas despachadas } \\
\hline $\begin{array}{lllllll}\text { Internas } & \ldots & \ldots & \ldots & \ldots & \ldots & \ldots\end{array}$ & $\cdots \cdots$ & 9.210 & 8.170 & - & 11,30 \\
\hline $\begin{array}{lllllll}\text { Externas } & \ldots & \ldots & \ldots & \ldots & \ldots & \ldots\end{array}$ & & 47.288 & 55.998 & + & 18,41 \\
\hline \multicolumn{6}{|c|}{ Consultas externas de puericultura y pediatría } \\
\hline Primera vez $\ldots \ldots \begin{array}{llll} & \ldots & \ldots\end{array}$ & $\ldots \ldots$ & 6.555 & 7.265 & + & 10.83 \\
\hline $\begin{array}{lllllll}\text { Segunda vez } & \ldots & \ldots & \ldots & \ldots & \ldots\end{array}$ & $\ldots \ldots$ & 15.126 & 20.245 & + & 33,84 \\
\hline Intervenciones quirúrgicas infantiles & $\ldots \ldots$ & 154 & 220 & + & 42,85 \\
\hline Numero de camaas adultas: 365 & & & & & \\
\hline Número de camas infantiles: & & & & & \\
\hline
\end{tabular}


CUADRO Y

CUADRO SINPOTICO DEL MOVIMIENTO CIENTICO-ASISTENCIAL

DEL INSTITUTO MATERNO INFANTIL

"CONCEPCION VILLAVECES DE ACOSTA"

1964 (Comparación con 1963)

\begin{tabular}{|c|c|c|c|c|}
\hline & 1963 & 1964 & & $\%$ \\
\hline Entradas $\ldots \ldots \ldots$ & 24.198 & 25.283 & + & 4,48 \\
\hline $\begin{array}{llllllll}\text { Estancias } & \ldots & \ldots & \ldots & \ldots & \ldots & \ldots & \ldots\end{array}$ & 93.167 & 105.145 & + & 12,86 \\
\hline Egresos $\ldots \ldots \ldots \ldots$ & 24.072 & 25.283 & + & 17,81 \\
\hline $\begin{array}{lllllllll}\operatorname{Partos} & \ldots & \ldots & \ldots & \ldots & \ldots & \ldots & \ldots & \ldots\end{array}$ & 16.247 & 17.751 & + & 9,26 \\
\hline Nacidos vivos $\ldots \ldots \ldots$ & 15.873 & 17.392 & + & 9,57 \\
\hline Nacidos muertos $\ldots \ldots \ldots$ & 520 & 557 & + & 7,12 \\
\hline Cesáreas $\ldots \ldots \ldots$ & 796 & 952 & + & 19,60 \\
\hline Aplic. de fórceps $\ldots \ldots \ldots \ldots$ & 1.800 & 1.466 & - & 18,56 \\
\hline Abortos atendidos $\ldots \ldots \ldots \ldots$ & 3.852 & 4.170 & + & 8,26 \\
\hline Defunciones maternas $\ldots \ldots \ldots$ & 37 & 35 & - & 5,41 \\
\hline Defunciones infantiles $\ldots \ldots \ldots \ldots$ & 536 & 543 & + & 1,31 \\
\hline Intervenciones varias $\ldots \ldots \ldots c c c c$ & 198 & 255 & + & 28,79 \\
\hline
\end{tabular}

Consultas prenatales:

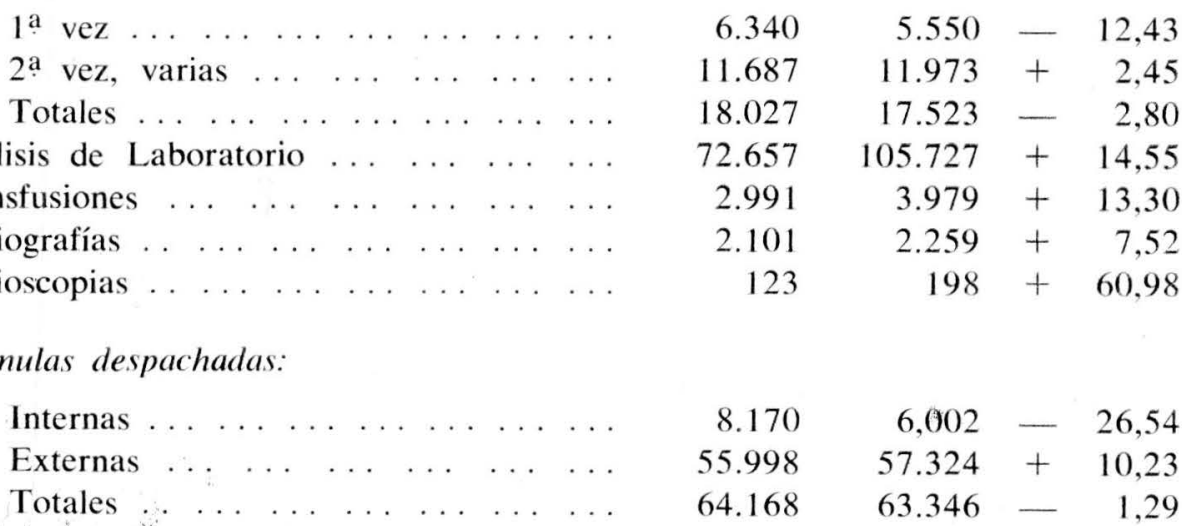

Consultas pediatría:

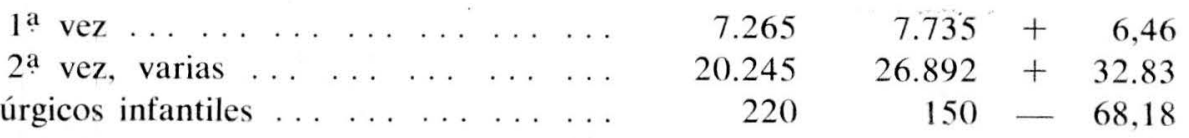


TABLA G

TOXEMIAS

1960:

$\begin{array}{lllllllr}\text { Partos } \ldots & \ldots & \ldots & \ldots & \ldots & \ldots & \ldots & 11.916 \\ \text { Toxémicas } & \ldots & \ldots & \ldots & \ldots & \ldots & \ldots & 438\end{array} \quad(\%=3,67)$

1961:

Parto

13.165

Toxémicas

$$
601(\%=4,56)
$$

1962:

Partos

Toxémicas

$575(\%=4,13)$

1963:

Partos

16.247

Toxémicas $280(\%=1,72)$

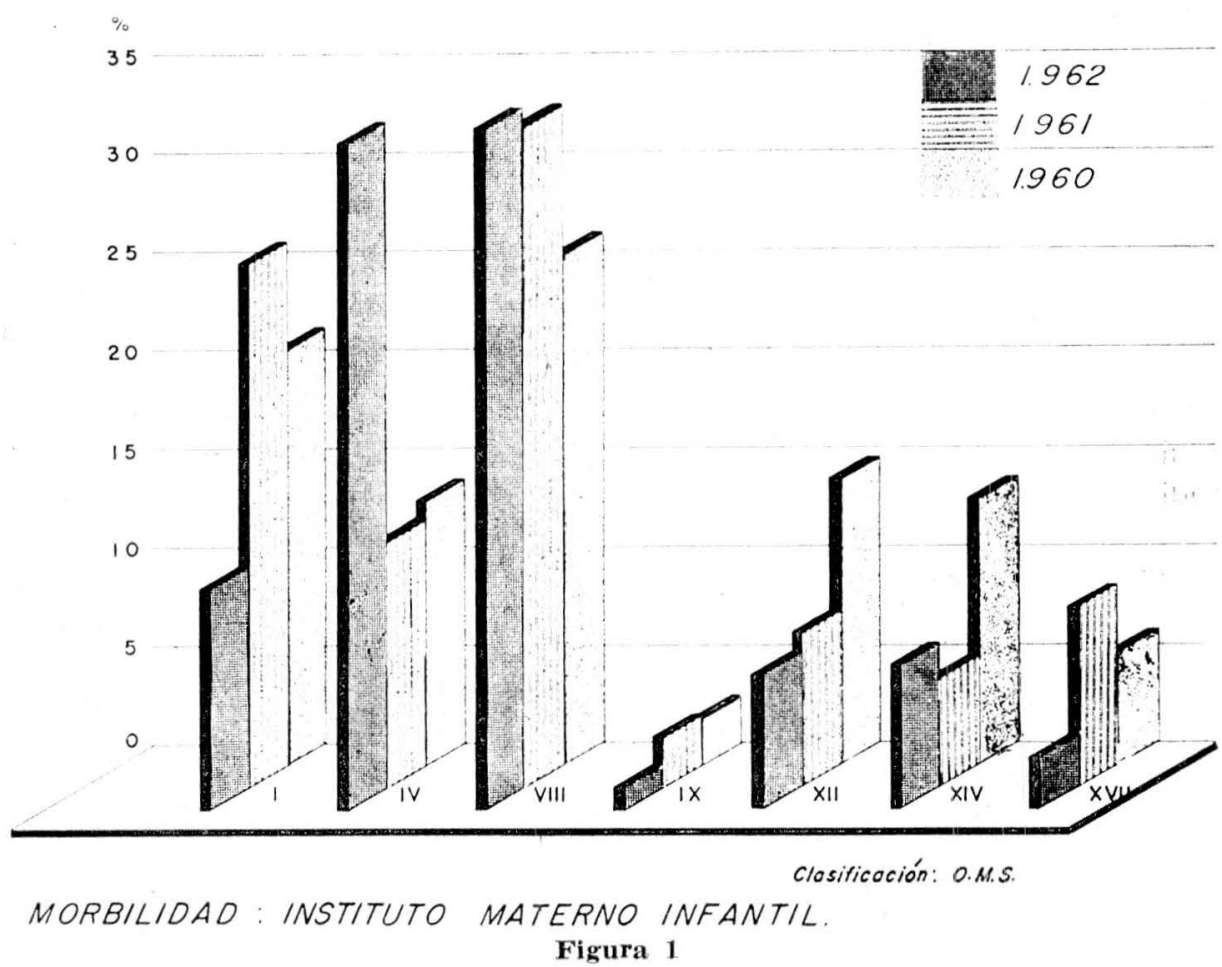




\section{OBSERVACIONES}

Instituto Materno infantil. 1964

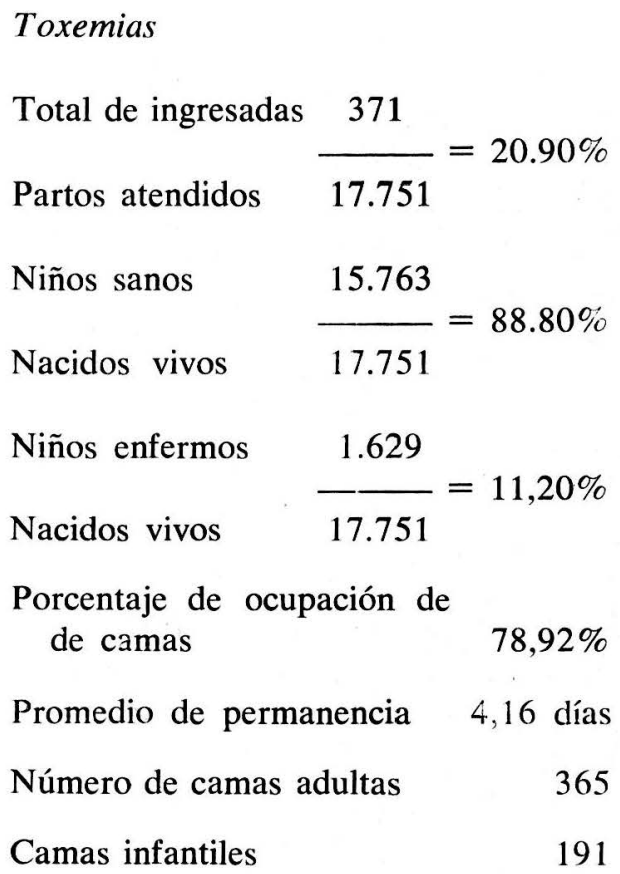


CUADRO $\mathrm{z}$

INSTITUTO MATERNO INFANTIL "CONCEPCION VILLAVECES DE ACOSTA"

SERVICIOS PEDIATRICOS

(Junio 1964)

ALBERTO AMARIS MORA, J e f a

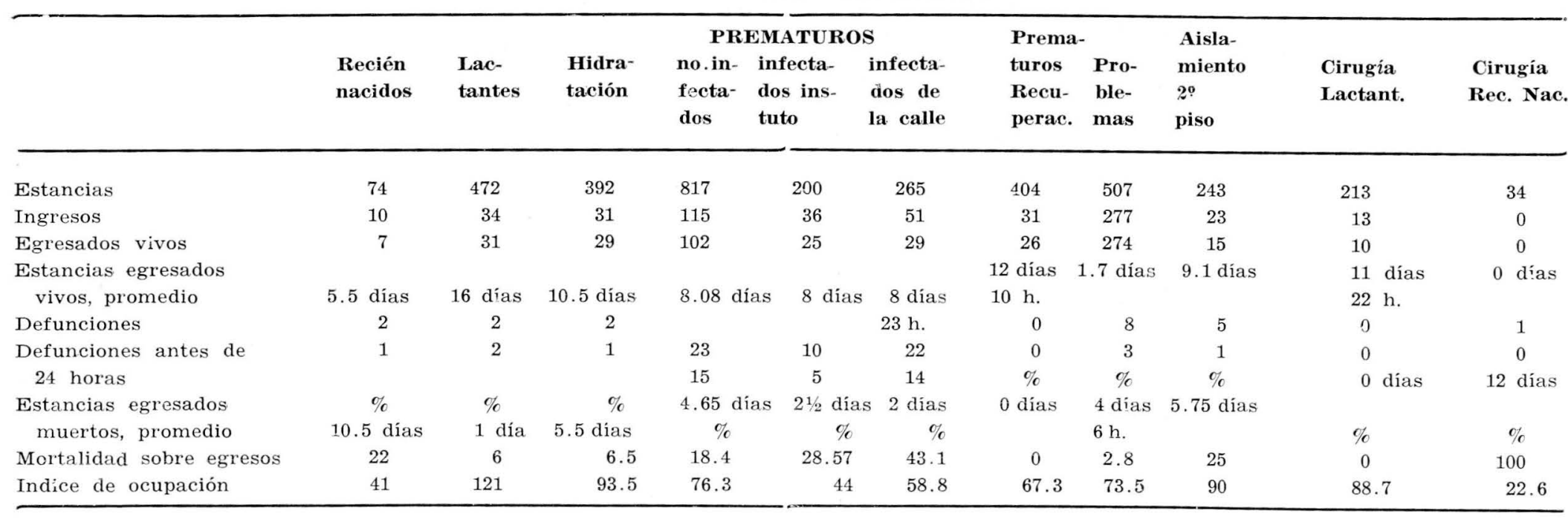




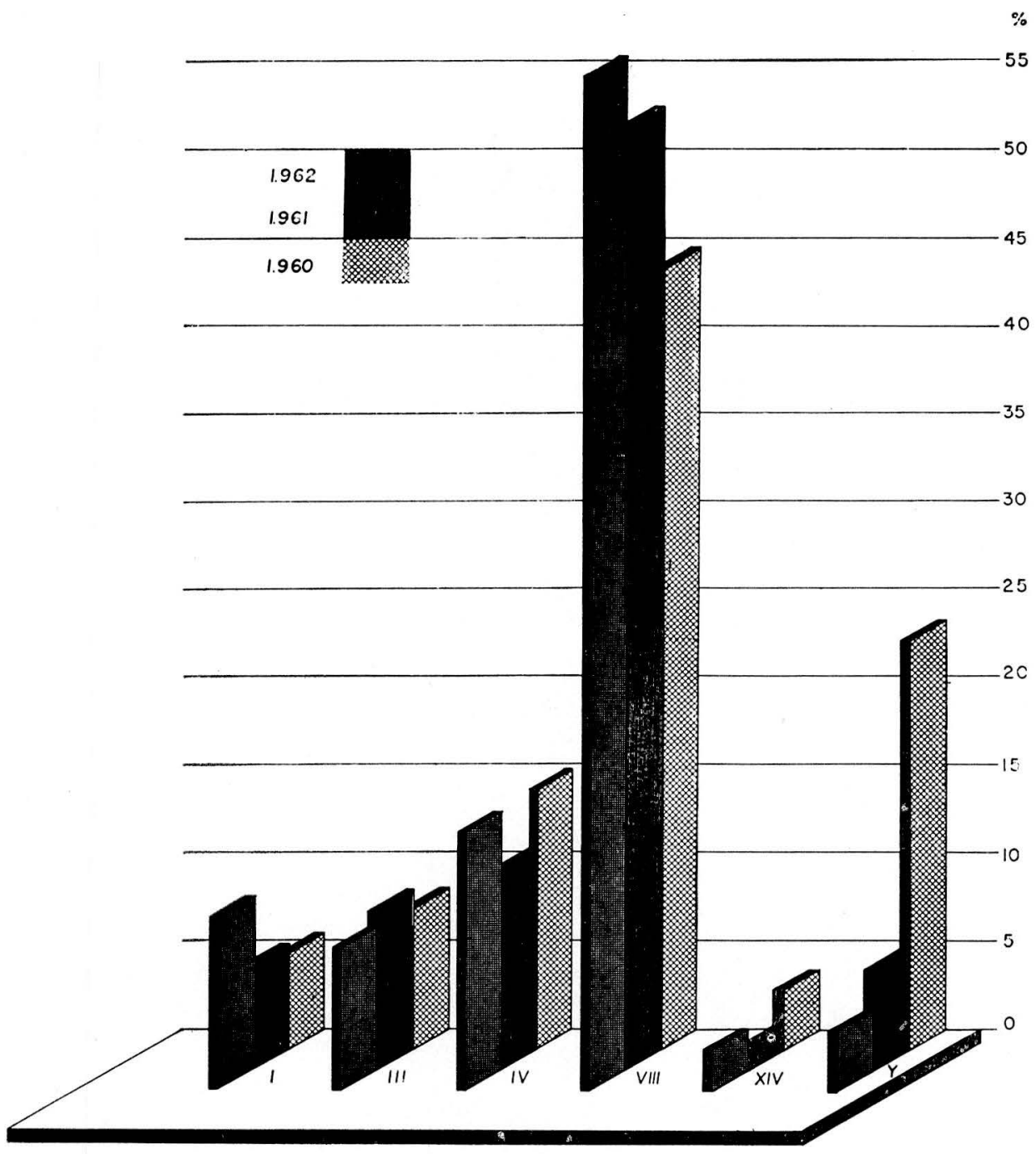

MORTALIDAD INFANTIL

Clasificación : O.M.S.

Figura 2 

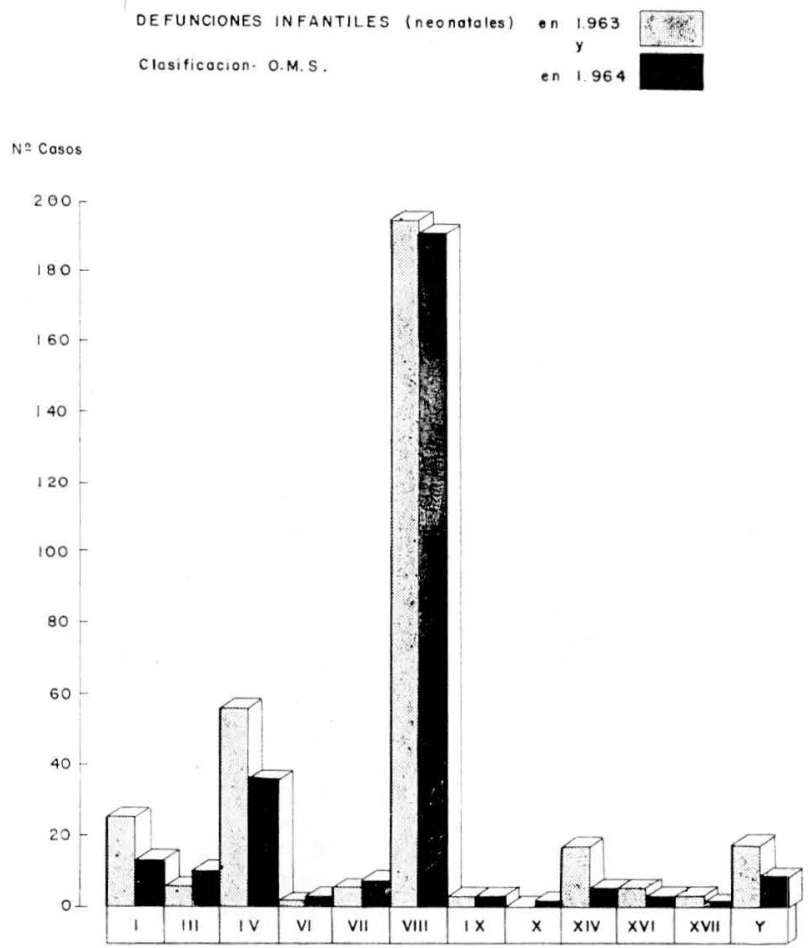

Figura 3
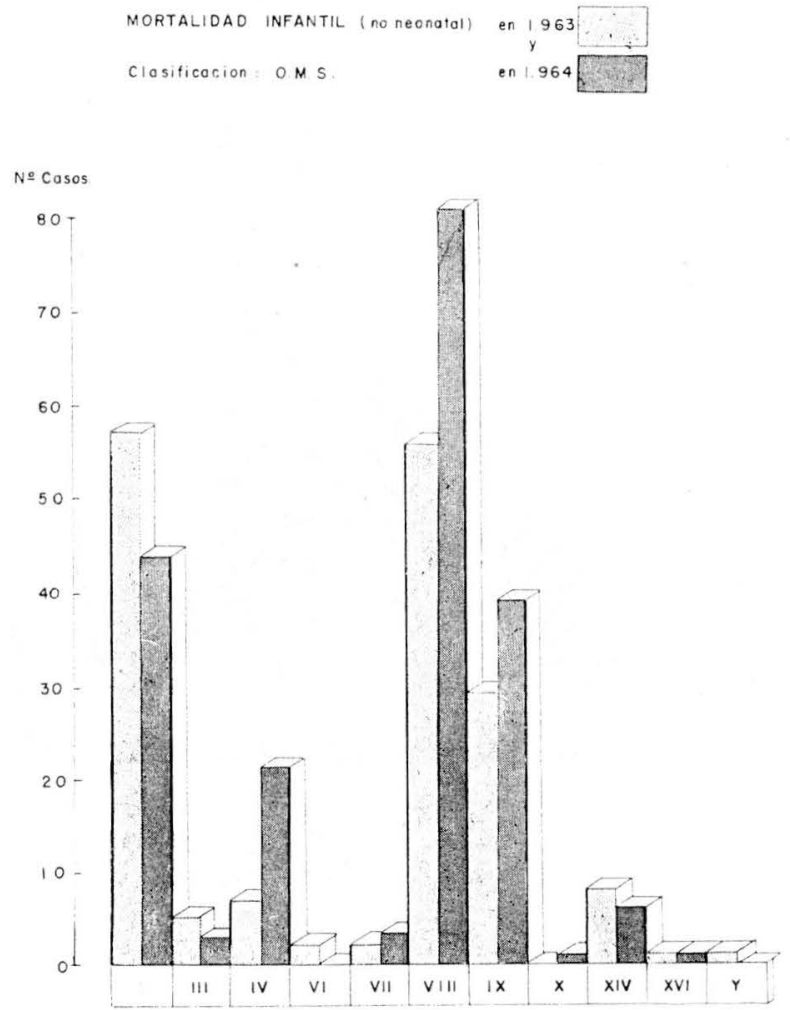

Figura 4 
Clasificacion. OM S

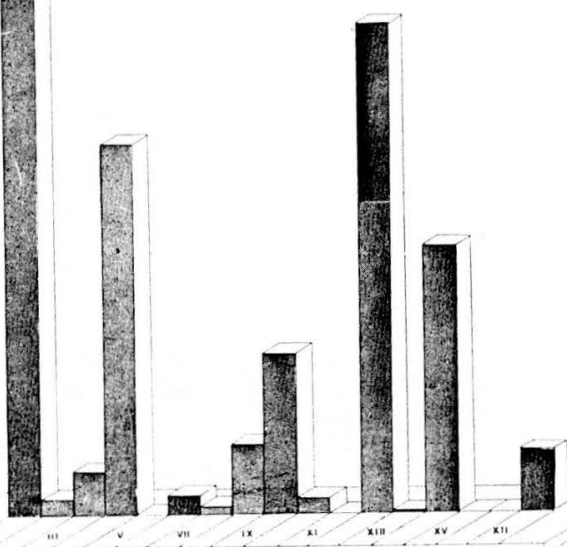

Figura 5

Morbitad Moterno (no obstétrica) on 1.964

ciasilicacion O.M.S

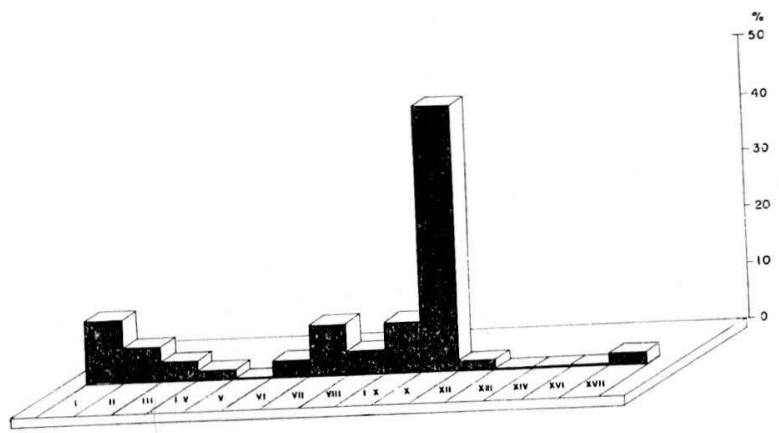

Figura 6 
Juiio-Agosto, 1965 Rev. Col. Obs. y Ginec.
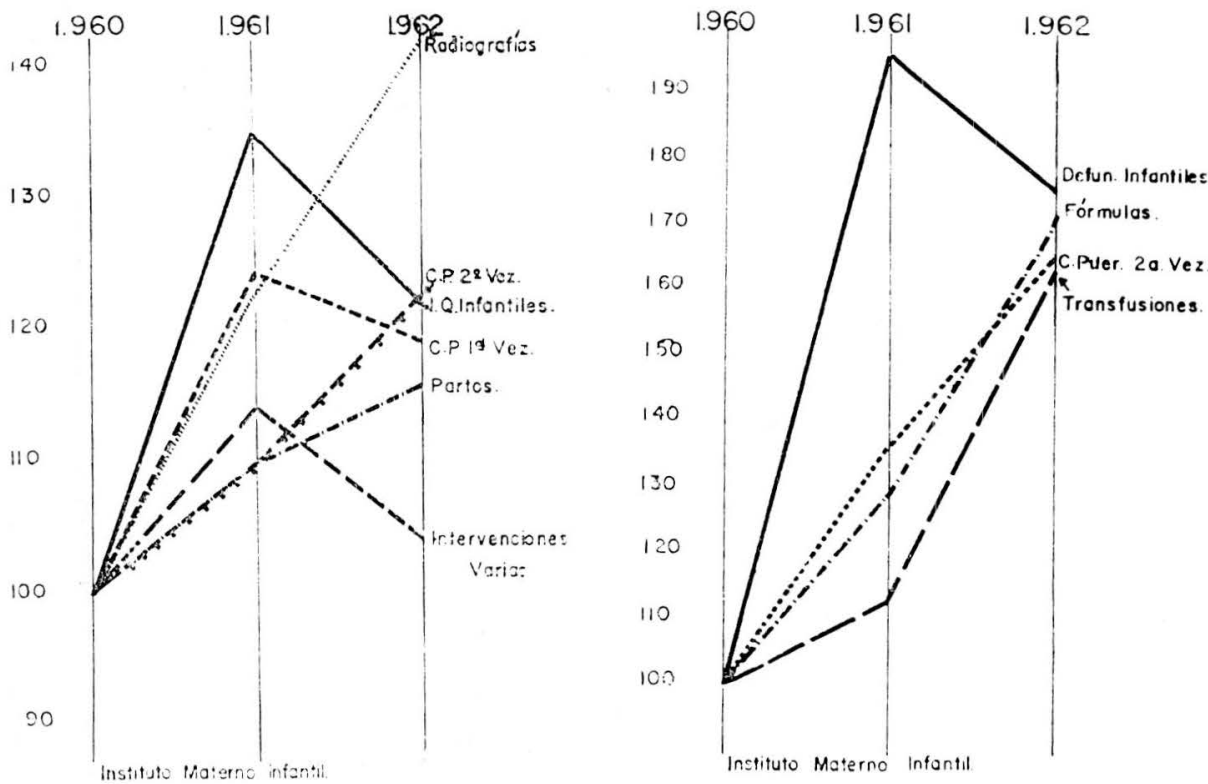

Figura 7
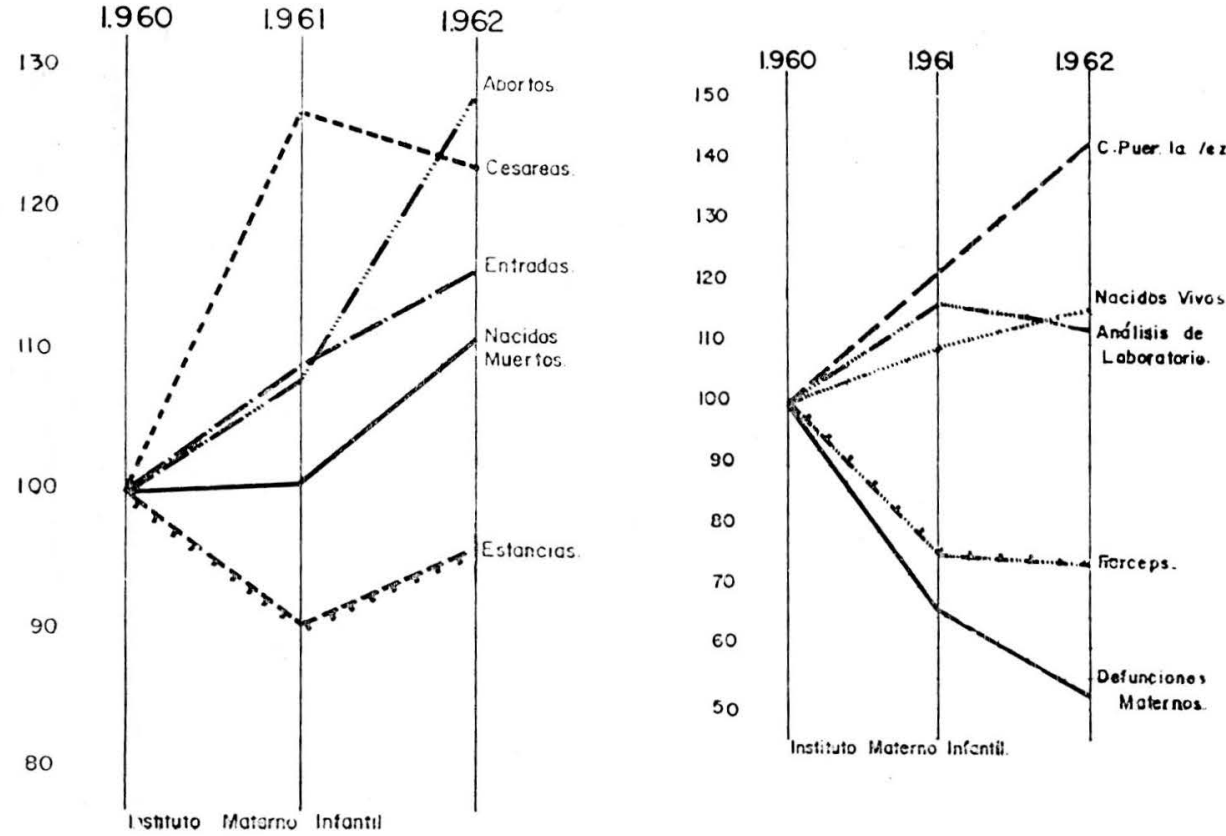

Figura 8 


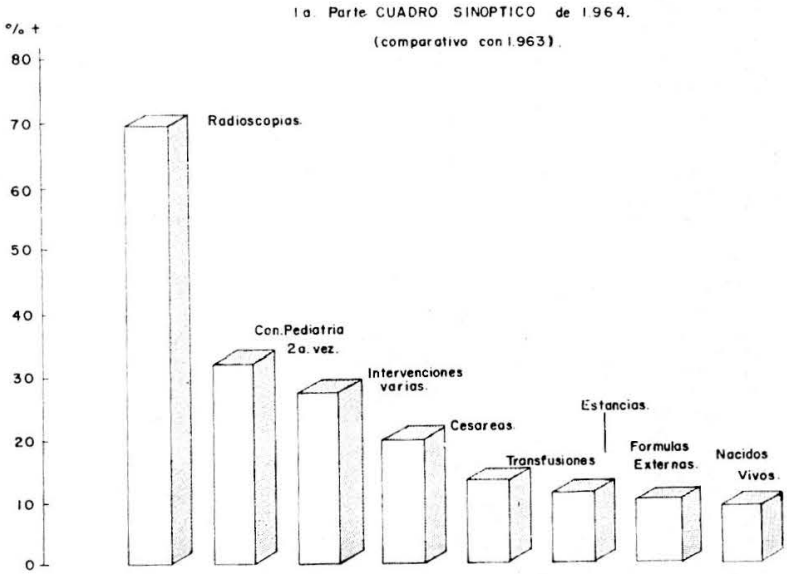

Figura 9

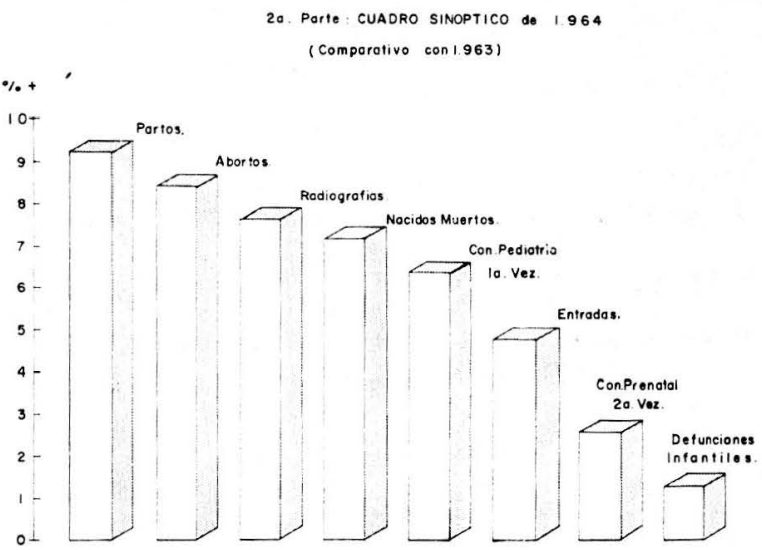

Figura 10 


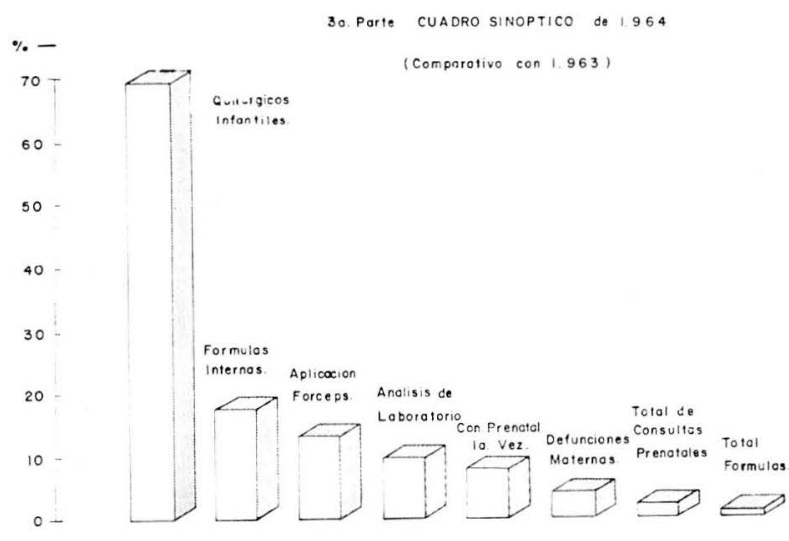

Figura 11

\section{BIBLIOGRAFIA}

AMERICAN MEDICAL ASSOCIATION: Standard Nomenclature of Diseases and Operations, Fifth edition. 3-81, 105482, 484-510, 517-610. The B'ackkinston Company. New York, Philadelphia, Toronto.

AUROUSSEAU P. y CHEVERNY R.: L' Hôpital de demain. Editions Masson, 1965, París.

LOPEZ-PICHOURON RAFAEL: La Estadística en el Hospital Colombiano Moderno. Tesis Universidad Javeriana. Octubre, 1962.

LOPEZ-RUIZ RAFAEL: Bioestadística simplificada y código internacional OMS (resumido). Revista del Hospital San Juan de Dios, Bogotá, II, 8; 324329. Año 1954. Revista del Hospital. Auge científico-asistencial en los establecimientos de San Juan de Dios. 33: 40. Vol. VI. Nọ 24, 1962. 
Morbilidad y Mortalidad en los Establecimientos Hospitalarios de San Juan de Dios, Bogotá, 1960, 62, 63. Tribuna Médica Vol, IV, № 164, noviembre 1964, 2-20. Ediciones Lerner, calle 14 Nọ 6-77, Bogotá.

Morbilidad y Mortalidad en los Establecimientos Hospitalarios de San Juan de Dios, Bogotá. 1964. Tribuna Médica. Vol. V. № 209. Octubre 1965. Ediciones Lerner, calle 14 № 6-77. Bogotá.

ORGANIZACION MUNDIAL DE LA SALUD (OMS): Manual de clasificación de Estadística Internacional de Enfermedades, traumatismos y causas de defunción, 7ạ Revisión, 3-43, 48-316. Ginebra, mayo, 1957. Indice de diagnóstico de hospitales y clasificcción de operaciones; 9-211, 212-273. Publicaciones Científicas Nọ 52, Washington D. C. mayo 1961.

QUIJADA CERDA OSWALDO: Atención organizada del enfermo y su documentación clínica y administrativa en el Hospital Moderno. 211-243. Central de Publicaciones. Casilla 38. Santiago de Chile. 